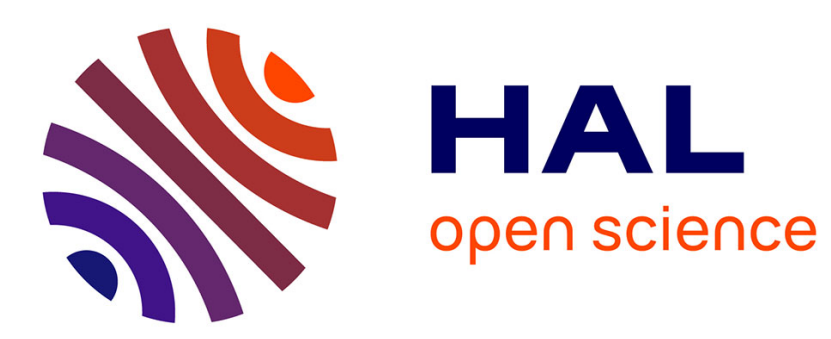

\title{
Generation of spherical vortex beams to trap large particles for enhanced axial force
}

D. Zhao, Jean-Louis Thomas, R. Marchiano

\section{To cite this version:}

D. Zhao, Jean-Louis Thomas, R. Marchiano. Generation of spherical vortex beams to trap large particles for enhanced axial force. Ultrasonics, 2021, 111, pp.106296. 10.1016/j.ultras.2020.106296 . hal-03160222

\section{HAL Id: hal-03160222 \\ https://hal.science/hal-03160222}

Submitted on 5 Mar 2021

HAL is a multi-disciplinary open access archive for the deposit and dissemination of scientific research documents, whether they are published or not. The documents may come from teaching and research institutions in France or abroad, or from public or private research centers.
L'archive ouverte pluridisciplinaire HAL, est destinée au dépôt et à la diffusion de documents scientifiques de niveau recherche, publiés ou non, émanant des établissements d'enseignement et de recherche français ou étrangers, des laboratoires publics ou privés. 


\title{
Generation of spherical vortex beams to trap large particles for enhanced axial force
}

\author{
Zhao D., ${ }^{\mathrm{a}, \mathrm{b}}$, Thomas J.-L. ${ }^{\mathrm{a},}$, Marchiano R. ${ }^{\mathrm{b}}$ \\ ${ }^{a}$ Sorbonne Université, CNRS, Institut des Nanosciences de Paris, INSP, F-75005 Paris, France \\ ${ }^{b}$ Sorbonne Université, CNRS, Institut Jean le Rond d'Alembert, d'Alembert, F-75005 Paris, France
}

\begin{abstract}
Recent studies have shown the possibility to manipulate small elastic spheres in 3D with a single-sided beam. Acoustical tweezers are very appealing because they provide a fine spatial control of the motion of a single particle in space. Their main limitations are due to the weak restoring axial force and improving this force is still a challenge. We show theoretically that the spherical vortex beams can trap large particles and enhance the axial force. Indeed, the special features of these unusual beams look like the bottle beams in optics. Nevertheless, their spatial complexity presupposes that they can be produced with sufficient precision. Therefore, attention is paid to the synthesis of the spherical vortices. A method based on the inverse filter method is proposed. It allows to synthesize them with a very good precision since the theoretical force is recovered experimentally with an error smaller that than $10 \%$. Then, the spherical vortices are used to trap polyethylene beads with radii between 500 and $590 \mu \mathrm{m}$. Experiments show that the radial trap is working while no beads have been trapped in the axial direction. This failure is analyzed in detail and is shown to be mainly due to sensitivity to the properties of the materials which influences the resonance modes of the elastic sphere. This study paves the way to the optimization of acoustical tweezers for the manipulation of large objects.
\end{abstract}

Keywords: Radiation pressure, Acoustical tweezers, single beam tweezers, spherical vortex beam, Resonating spherical target

2010 MSC: 00-01, 99-00

${ }^{\star}$ Fully documented templates are available in the elsarticle package on CTAN

URL: WwW. elsevier. com (Zhao D.) 


\section{Introduction}

Contactless manipulation using radiation pressure is widely used in biological, chemistry and medical applications. Among available tools, optical tweezers [4] stand out for their selectivity, the ability to pick up a selected particle among others, and accuracy. They have been used to trap various kinds of targets such as cells, viruses and molecules [40, 1, 24, 3, 36]. Meanwhile, the shortcomings of optical tweezers: the photo-damage due to photochemistry [9] and unavoidable heating [23] with highly focused optical beam restrict the range of force in practical situations (from 0.1 to $100 \mathrm{pN}$ [30]). Magnetic tweezers are safe for biological samples but have their limitations in terms of forces and trap stiffness. In this context, the noninvasive and deep-tissue-penetration properties of ultrasound are very attractive for complex manipulations in biological and biomedical domains.

Acoustical traps based on standing waves were proved to be feasible and can provide stable traps for spheres, droplets, living animals [39, 43, 42]. In these setups, the standing waves are made with a single transducer and a mirror or two transducers in opposite directions. Hence the targets must be located in between and thus forbid three-dimensional manipulation in many practical situations. Moreover, a single location of equilibrium is achieved only if the cavity length is one half of wavelength. Indeed, all pressure nodes (or anti-nodes depending on the object density and compressibility) act as potential traps. Such a constraint means that submillimetric wavelengths much larger than the micrometric targets are usually used even in microfluidic channels. Standing wavefields are of significance in collective manipulation of multiple objects or for particle sorting. On the contrary, single-beam optical tweezers achieve selective traps with a single position of equilibrium of size comparable to the particles. Diffraction in the far-field limits the focal spot to a size larger than about one wavelength, therefore achieving acoustical tweezers with optimal selectivity means trapping object of about the size or larger than one wavelength. Moreover, they afford three-dimensional manipulations with nanometer accuracy. Regarding the advantage of three-dimensional manipulation, single beam acoustical tweezers consisting of single-sided transducer or transducers array are developed [5, 11, 21]. For such single-sided configurations, the most important parts are the beamforming and transducer design. To date, different beamforming of single beam acoustical tweezers have been investigated: focused Gaussian beam [34, 19, 20], Bessel beam [15, 13, 26, 12], fraxicon beam [22] and focused vortex beam [7]. 
The major challenge of acoustic single beam trapping is to achieve the axial restoring force since there to manipulate polyethylene beads (Sec. 3.2). Finally, the analysis of the resonances of large spheres, as well as their influence on the trapping, are discussed.

\section{Methods}

\subsection{Theoretical predictions of the forces exerted by a spherical vortex on an elastic sphere}

Spherical vortex, as suggested by its name, is a vortex beam whose associated pressure field has a spherical pattern at the central beam core. As is shown in Fig. 1(b), the high amplitude has a 'candy' 
form, the zero amplitude region is like a spherical 'candy' wrapped up by the high-pressure field. This kind of vortex has a high-amplitude gradient both radially and axially and forms a spherical trapping zone with zero amplitude at the center. Compared to a focused vortex beam with the same topological charge (Fig. 1(a)p, the spherical vortex has a higher pressure gradient in the wave propagation direction and a larger trapping zone (Fig. 1(b)) . According to this configuration of the pressure field, the spherical vortex is supposed to be able to trap large spheres radially and axially (with radius $a \simeq 0.5 \lambda$ ). 

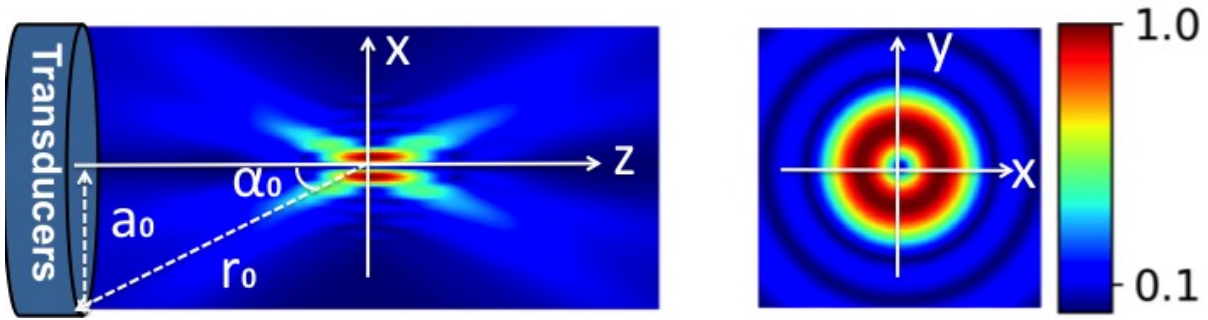

(a) Theoretical pressure field of a focused vortex
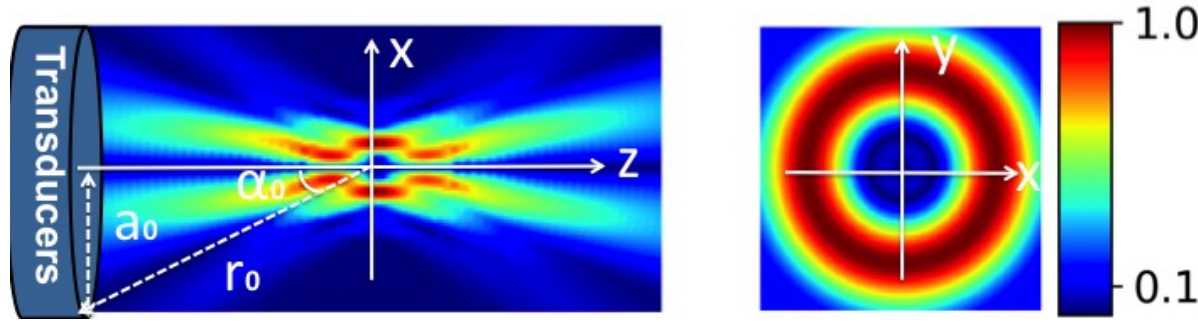

(b) Theoretical pressure field of a spherical vortex
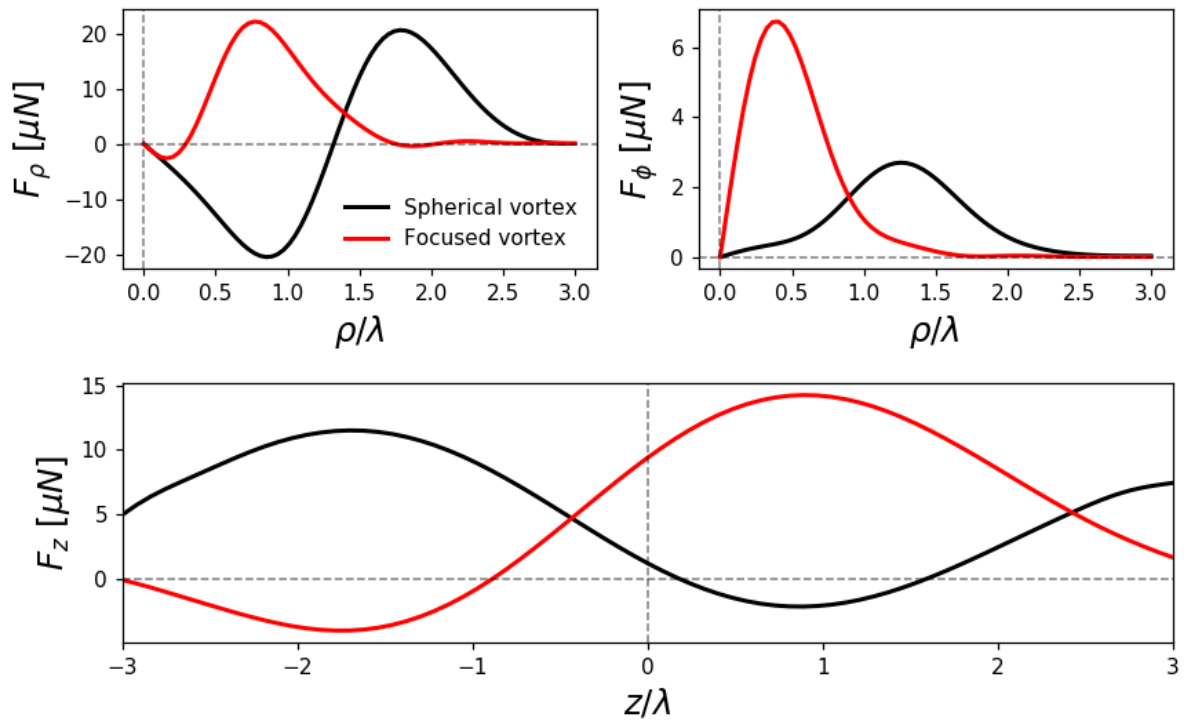

(c) Theoretical radiation forces

Figure 1: (Color online) Modulus of the complex pressure field at frequency $f_{0}=1.2 \mathrm{MHz}$ for (a) a focused vortex beam and (b) a spherical vortex beam with topological charge $m^{\prime}=1$, radial degree $l=8$ generated with a concave spherical transducer: aperture angle $\alpha_{0}=49^{\circ}$, geometrical focus $r_{0}=7.5 \mathrm{~cm}$, the spherical basis is centered at the focus of the incident beam; (c) Radiation forces on a polyethylene sphere with radius $a=0.45 \lambda$ exerted by a focused vortex beam with topological charge $m^{\prime}=1$ and aperture angle $\alpha_{0}=49^{\circ}$ and a spherical vortex beam of same topological charge and aperture angle with radial degree $l=8$.

In a spherical basis, the incident wavefield can be decomposed with spherical functions and described 
by the incident beam shape coefficients (BSC) $A_{n}^{m}$ :

$$
p_{i}(r, \theta, \phi, t)=p_{a} \sum_{n=0}^{+\infty} \sum_{m=-n}^{n} A_{n}^{m} j_{n}(k r) Y_{n}^{m}(\theta, \phi) \exp (-i \omega t)
$$

where $p_{a}$ is the characteristic amplitude, $j_{n}$ is the spherical Bessel function and $Y_{n}^{m}$ are the spherical harmonics.

The BSC for a focused vortex are [5]:

$$
A_{n}^{m}=\delta_{m, m^{\prime}} N_{n}^{m^{\prime}}\left(k r_{0}\right)^{2} h_{n}^{(1)}\left(k r_{0}\right)(-1)^{n+m^{\prime}} \int_{\cos \alpha_{0}}^{1} P_{n}^{m^{\prime}}(x) d x
$$

and for a spherical vortex:

$$
A_{n}^{m}=\delta_{m, m^{\prime}} 4 \pi N_{n}^{m^{\prime}}\left(k r_{0}\right)^{2} h_{n}^{(1)}\left(k r_{0}\right)(-1)^{n+l} \int_{\cos \alpha_{0}}^{1} \tilde{P}_{l}^{m^{\prime}}(x) P_{n}^{m^{\prime}}(x) d x
$$

where the coefficients $N_{n}^{m^{\prime}}$ are:

$$
N_{n}^{m^{\prime}}=\sqrt{\frac{(2 n+1)}{4 \pi} \frac{\left(n-m^{\prime}\right) !}{\left(n+m^{\prime}\right) !}}
$$

Function $h_{n}^{(1)}$ is the spherical Hankel function of the first kind, the wavenumber is $k=2 \pi / \lambda$, functions $P_{n}^{m}$ and $\tilde{P}_{l}^{m^{\prime}}$ are the associated Legendre polynomials and associated Legendre polynomials normalized to unity, respectively, $x=-\cos (\theta)=\cos (\alpha)$, with $\alpha=\pi-\theta,(r, \theta, \varphi)$ the spherical coordinates. With $\delta_{m, m^{\prime}}$ the Kronecker delta, $m^{\prime}$ the topological charge of the vortex and $\alpha_{0}, a_{0}, r_{0}$ are respectively the aperture angle, the radius of the transducer array and the focal distance as illustrated in Fig. 1(b). Comparing the beam shape coefficients of the two vortex beams, the spherical vortex is obtained by using the associated Legendre polynomial to tailor the focused vortex beam. Thus, for a spherical vortex of topological charge $m^{\prime}=1$, the shape is mainly determined by the aperture angle $\alpha_{0}$ and the degree $l$ of the associated Legendre polynomial $\tilde{P}_{l}^{m^{\prime}}(x)$ in Eq. 3 . Among these factors, the aperture angle $\alpha_{0}$ is limited by the directivity pattern of the transducers and the lens used to focus the field. The maximum angle of the aperture is generally fixed by the experimental setup (for instance in our experimental system, it is $49^{\circ}$ ). Thus, to achieve the desired forces acting on an elastic sphere, the optimal topological charge $m^{\prime}$ and the degree $l$ should be chosen. First, the degree $l$ of the associated Legendre polynomial $\tilde{P}_{l}^{m^{\prime}}(x)$, is chosen 
to have an amplitude that varies smoothly in the range of $x=\left[\cos \alpha_{0}, 1\right]$ and gently drops near zero at $\cos \alpha_{0}$ to minimize the secondary lobes in the radiated field that would be induced by a sharp truncation. As depicted in Fig. 2, the topological charge $m^{\prime}$ is fixed at $1, \tilde{P}_{l}^{m^{\prime}}(x)$ as a function of $\alpha$ is plotted for different degrees $l$. According to the figure, the degree $l=4$ and $l=8$ are both close to zero at $\alpha_{0}=49^{\circ}$. Actually, $l=4$ is closer to zero than $l=8$. However, comparing the pressure field for $l=4$ and $l=8$ (Fig. 3), we find that in plane $(x, z)$, the axial pressure gradient for $l=8$ is larger than $l=4$. And in plane $(x, y)$, the size of the trapping region (the blue zone inside the high intensity ring) for $l=8$ is also larger than $l=4$. Therefore, a compromise should be made to obtain the optimal beam shape. As our aim is to trap large particles, which need a large trapping zone and a high pressure gradient in the axial direction, the degree $l=8$ is the best suited with $m^{\prime}=1$.

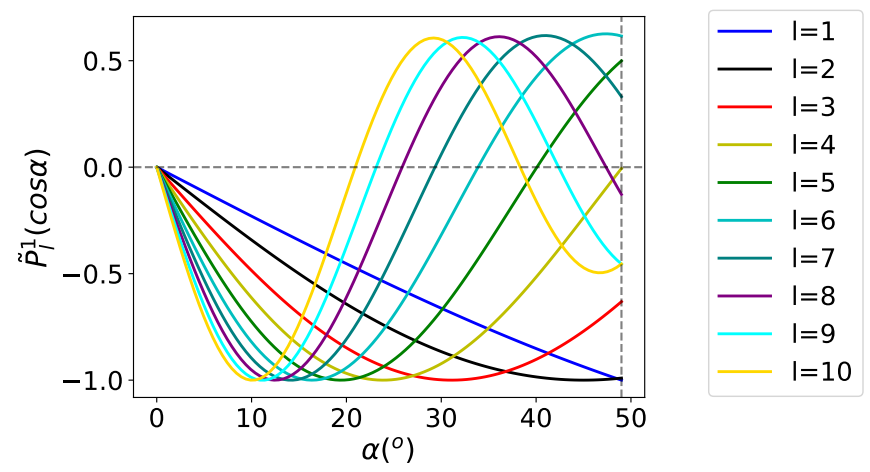

Figure 2: (Color online) Normalized associated Legendre polynomial $\tilde{P}_{l}^{m^{\prime}}(\cos \alpha)$ as a function of $\alpha$. A vertical grey line is drawn to mark $\alpha_{0}=49^{\circ}$. 

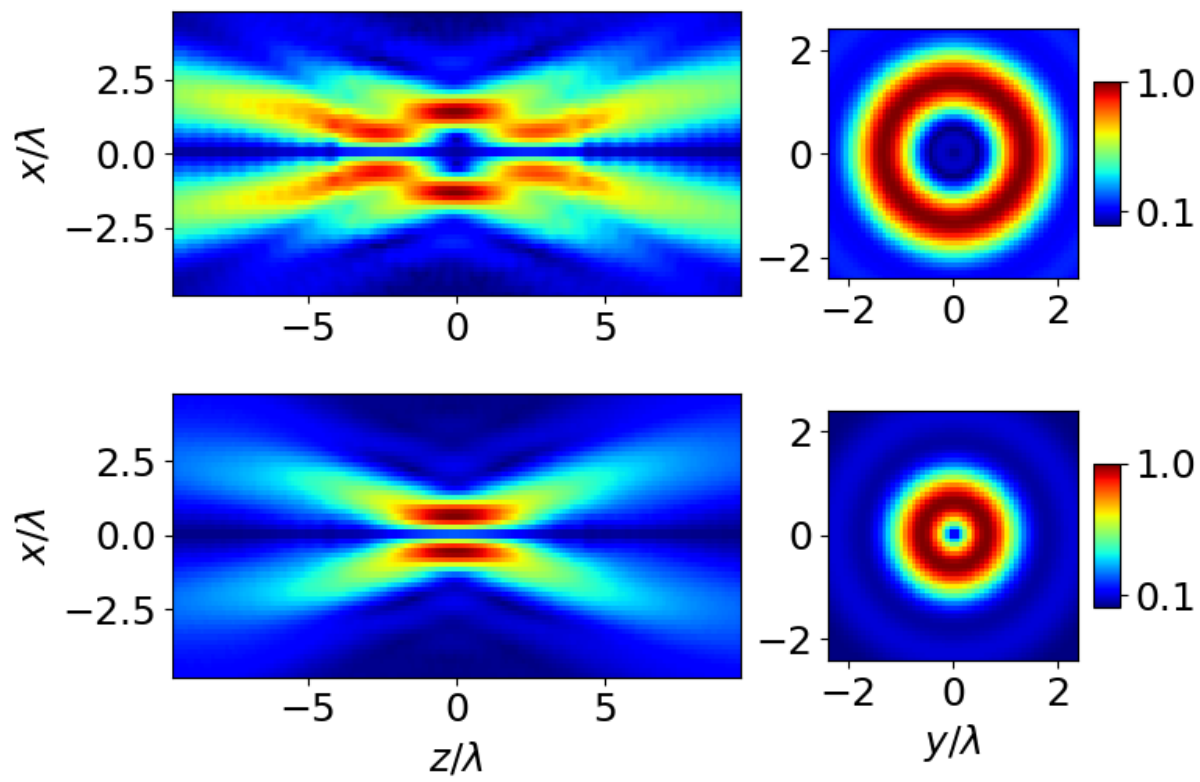

Figure 3: Modulus of the pressure field in plane $(x, y)$ and $(x, z)$ with $l=8$ (top) and $l=4$ (bottom).

The trapping effect of a focused vortex and a spherical vortex beam are assessed by computing the radiation forces. To do so, an efficient model established recently [6] is applied. According to this model, the three-dimensional radiation force acting on an elastic sphere in an arbitrary wavefield can be computed using the BSC and scattering coefficients. As illustrated in Fig. 1(c), a polyethylene bead (density $\rho=1000 \mathrm{~kg} / \mathrm{m}^{3}$, transverse velocity $c_{t}=1000 \mathrm{~m} / \mathrm{s}$, longitudinal velocity $c_{l}=2400 \mathrm{~m} / \mathrm{s}$ ) with radius $a=0.45 \lambda$, immersed in water $\left(\rho_{0}=1000 \mathrm{~kg} / \mathrm{m}^{3}, c_{0}=1480 \mathrm{~m} / \mathrm{s}\right)$ can be trapped by the forces exerted by a spherical vortex but not by a focused vortex beam at frequency $f_{0}=1.2 \mathrm{MHz}$. Consequently, by tailoring the spherical vortex with proper topological charge and radial degree, it is possible to trap a large elastic sphere while it is not possible with a focused vortex. 

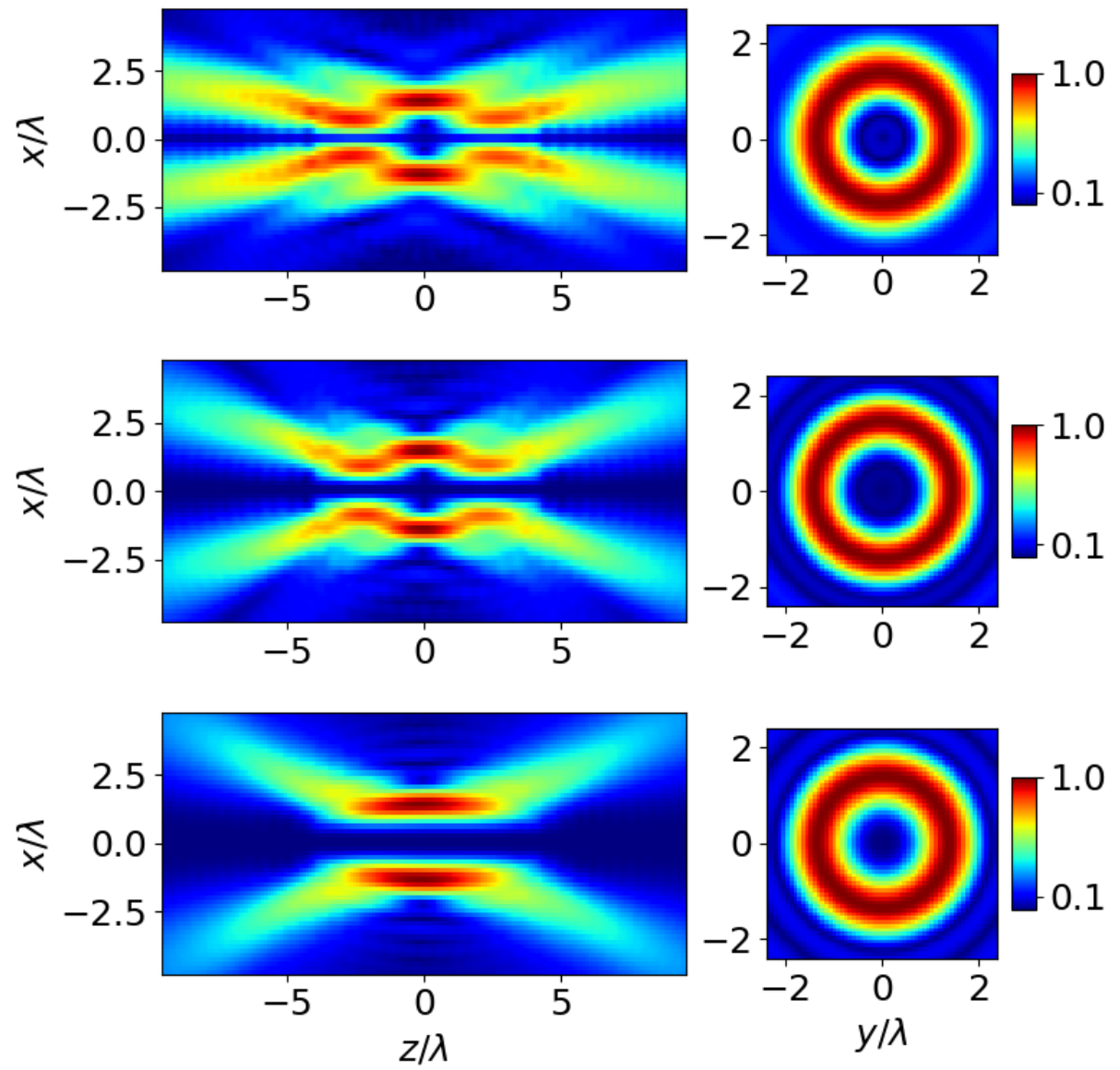

Figure 4: (Color online) Modulus of the pressure field in plane $(\mathrm{x}, \mathrm{y})$ and $(\mathrm{x}, \mathrm{z})$ with different topological charges: $m^{\prime}=1$, $m^{\prime}=2, m^{\prime}=3$.

Regarding the topological charge $m^{\prime}$, we have compared the amplitude of the pressure field with 100 $m^{\prime}=1, m^{\prime}=2, m^{\prime}=3$ in plane $(x, y)$ and $(x, z)$ (see Fig. 4). In plane $(x, y)$, the high-amplitude ring keeps almost the same shape and the area of zero amplitude has a radius of about $0.5 \lambda$. Meanwhile, the pressure gradient in plane $(x, z)$ varies a lot from $m^{\prime}=1$ to $m^{\prime}=3$. When $m^{\prime}=1$, the high amplitude has a 'candy' form, the zero amplitude region is like a spherical 'candy' wrapped up by high pressure field (see Fig. 4). By increasing $m^{\prime}$ to 2 , the zero amplitude region remains roughly spherical at the center, but the high pressure surrounding it begins to open at the two sides. For $m^{\prime}=3$, the zero amplitude region turns completely into a cylindrical shape. These variations of the beam shape change the pressure gradient and therefore, intuitively impacts the radiation forces. Computing the forces varying the charge $m^{\prime}$ from 1 to 3 , the amplitude of the axial force decreases as a result of the decrease of the pressure field 
amplitude. For $m^{\prime}=3$, no axial trap is predicted due to the lack of pressure gradient at the center of the

to the greatest pressure gradient. Therefore, the topological charge $m^{\prime}=1$ and radial degree $l=8$ are selected to generate a spherical vortex with a good capability for trapping the target sphere.

Note that, in this study the scattering between several particles is not taken into account and we are considering the scattering of only one particle in the model. Indeed, the goal is to improve the spatial selectivity of the tweezers and as a corollary to minimize the number of particles insonified. Nevertheless, if more than one particle are initially located in the spatially focused trap, these particles may reach configurations where multiple scattering may be of importance and equilibrium configurations with several particles have already been observed [8]. For these cases, resonances may appear in the wave scattering process of bouncing back and forth between the particles. Theoretical modeling of these cases is still an open issue.

\subsection{Experimental setup}

The experimental setup is shown in Fig. 5. An array of 127 piezoelectric transducers (Vermon, France) with a central frequency at $1.2 \mathrm{MHz}$ is held by a vertical support in a large water tank. The corresponding wavelength is $\lambda=1.23 \mathrm{~mm}$ in water at $20^{\circ} \mathrm{C}$ where the speed of sound is about $1480 \mathrm{~m} / \mathrm{s}$.

25 The surface of each transducer is a hexagon of $62.82 \mathrm{~mm}^{2}$ and they fill a spherical cap with a focal distance equal to $45 \mathrm{~cm}$. The total aperture of the transducer array is $110 \mathrm{~mm}$. A high numerical aperture bi-concave lens machined in PMMA allows to reduce the focal distance to about $6 \mathrm{~cm}$ (see Fig. 5). Each transducer is driven independently by a multichannel electronic (Lecoeur, France). The 127 electric signals used to synthesize the ultrasonic beam are computed by inverse filter method (see section 3.1). A very thin polyethylene film (with thickness $\approx 15 \mu \mathrm{m}$ ) that ensures minimal acoustic reflection is stretched on a bracket and is mounted on a three-axis motorized positioning system. The motors (ILS250, Newport) are powered and controlled by a remote command (Newport, MM-4006) with an accuracy of $5 \mu \mathrm{m}$. Behind an optical window, an optical zoom image the area of interest and the image is recorded by a CMOS camera (Pointgrey Flea3). At the bottom of the vertical support, four wheels are fixed to move the array of transducers. The pressure field is measured with a hydrophone of $0.2 \mathrm{~mm}$ diameter (Precision Acoustics Ltd, UK). 
Two kinds of polyethylene spheres were tested in our experiments: spheres with radii between $500 \mu \mathrm{m}$ and $590 \mu \mathrm{m}(0.4 \lambda-0.47 \lambda)$ with density $1000 \mathrm{~kg} / \mathrm{m}^{3}\left(990 \mathrm{~kg} / \mathrm{m}^{3}-1010 \mathrm{~kg} / \mathrm{m}^{3}\right)$ and $960 \mathrm{~kg} / \mathrm{m}^{3}$ $\left(960 \mathrm{~kg} / \mathrm{m}^{3}-980 \mathrm{~kg} / \mathrm{m}^{3}\right)$. The transverse and longitudinal velocities are estimated to be $: c_{t}=1000 \mathrm{~m} / \mathrm{s}$ and $c_{l}=2400 \mathrm{~m} / \mathrm{s}$ [31, 32].

Figure 5 shows the experimental setup in a configuration where the array of transducers is on the top oriented downwards. A second experimental configuration has also been used where the array of transducers is at the bottom and is oriented upwards. This second configuration is used to trap beads with density $960 \mathrm{~kg} / \mathrm{m}^{3}$. In this case the polyethylene film prevents the bead from rising up.

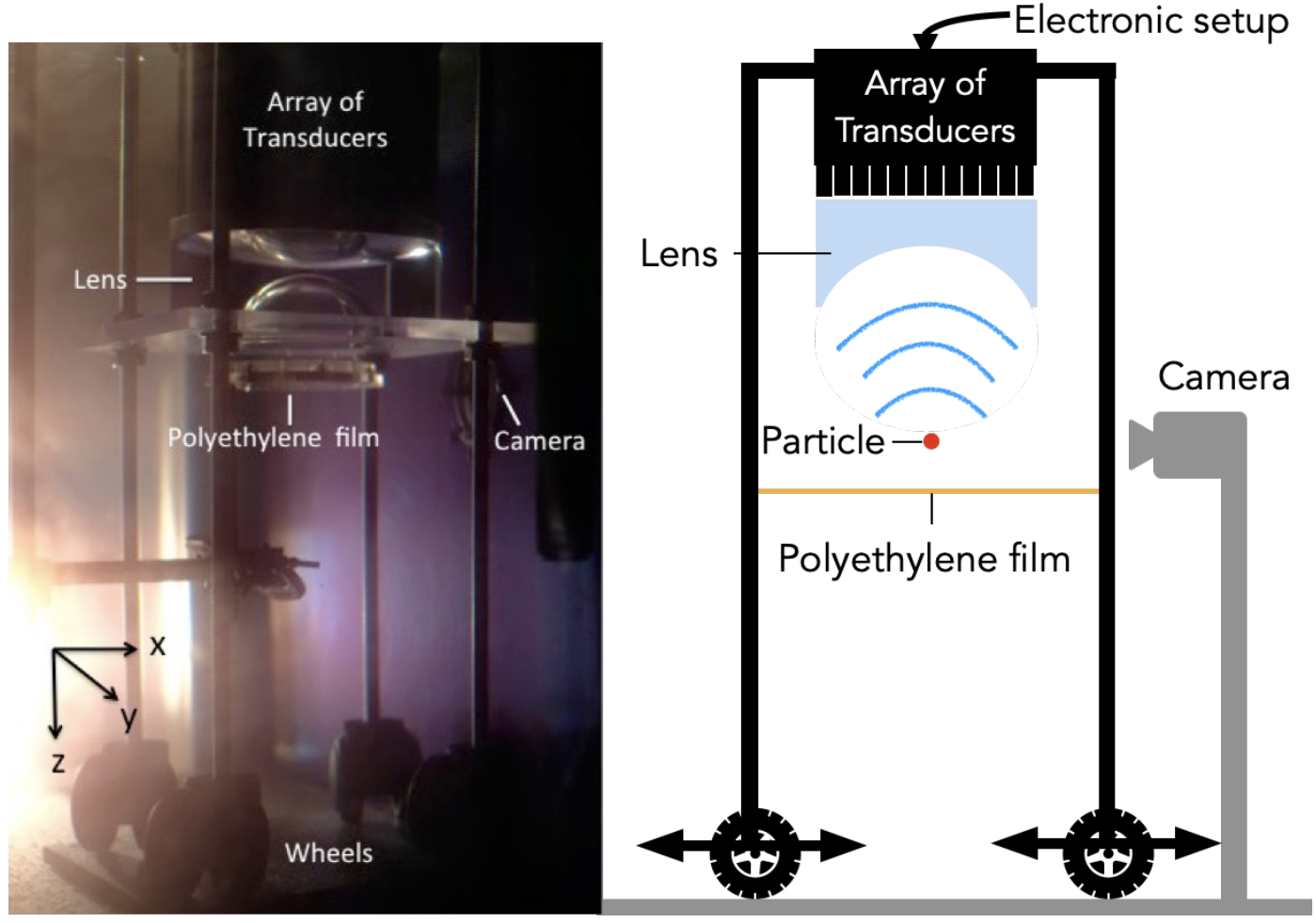

Figure 5: (color on line)Experimental setup: an array of transducers is positioned vertically with an acoustic lens to help the focusing; a polyethylene film is stretched on a bracket that is connected to a motorized positioning system.

\section{Experimental results}

\subsection{Experimental synthesis of a spherical vortex}

According to the analysis of section 2.1, a spherical vortex with topological charge $m^{\prime}=1$, radial degree $l=8$ is chosen to trap the elastic spheres. An accurate synthesis of the wavefield is required to 
achieve the desired trapping forces, especially the axial restoring force. With an array of transducers,

multiple methods can be applied to synthesize the desired acoustic field, including time reversal [14], inverse filtering [38, 37] and integrative angular spectrum technologies [41]. Among these methods, inverse filtering is a very general technique for analyzing and synthesizing complex signals that propagate through an arbitrary linear medium. This method provides the optimal input signals to get a target wavefield. It consists of three steps: the first is recording the propagation operator of the propagation medium which completely characterizes the medium and the geometry of the experiment; the second is calculating the inversion of the propagation operator; and finally computing the optimal input for the transducer array.

We consider $N$ control points whose positions are distributed in the volume of the medium. Let $f_{j}(t)$ be the signal received at the $j$ th control point $(1 \leqslant j \leqslant N)$ and $e_{i}(t)(1 \leqslant i \leqslant M)$ the signal emitted by the $i$ th transducer. As the medium can be thought as a linear and time invariant system, these two signals are related by:

$$
f_{j}(t)=\sum_{i=1}^{M} h_{j i}(t) * e_{i}(t),
$$

where $h_{j i}(t)$ is the impulse response between transducer $i$ and control point $j$. The symbol '*' denotes the convolution product. In the Fourier domain, this equation is transformed into a matrix/vector product: $\underline{F}(\omega)=\underline{\underline{H}}(\omega) \underline{E}(\omega)$, where $\underline{\underline{H}}$ is the frequency response of the system (size: $N \times M$ ), which describes the propagation of signals from the array of transducers to the control points and thus is called the propagation operator. The vectors $\underline{E}(\omega)$ (length: $M$ ) and $\underline{F}(\omega)$ (length: $N$ ) contain the spectra of the transmitted and received signals respectively. We aim to find the optimal signals to send $(\underline{E})$ by the array of transducers to generate the target field $\underline{F}$ on the control points after propagation. A naive approach consists in inverting directly this relation. However, the matrix $\underline{\underline{H}}$ is not square and cannot be inverted directly. Instead, the pseudo inverse is computed with a singular value decomposition, which allows adding a step of regularization through the choice of a cut-off in the singular values. Then, it is straightforward to get the emission vector to synthesize the target field $\underline{F}(\omega)$ :

$$
\underline{E}(\omega)=\underline{\underline{\hat{H}}}^{-1}(\omega) \underline{F}(\omega)
$$


with $\underline{\underline{\hat{H}}}^{-1}(\omega)$ the regularized inverse propagation operator.

The spatial distribution of the control points is the key parameter determining the efficiency of the inverse filter method. To get a propagation operator that completely characterize the medium, it is essential to over-sample the field in the area of interest. Among various trial distributions, we found that a regular distribution with 2500 points located in the focal plane $(x, y)$ gives the best result. For this distribution, the sampling points are evenly distributed on a square surface with side length $6 \mathrm{~mm}(4.9 \lambda)$ and with a step of $0.14 \mathrm{~mm}(0.11 \lambda)$. At the center, inside a square of side length $2 \mathrm{~mm}(1.62 \lambda)$, we refined the mesh and the step is decreased to $0.1 \mathrm{~mm}(0.08 \lambda)$. This small step is required to properly sample the pressure field which varies rapidly in the center (see Fig. 6(b)).

Once the emission vector is obtained, the associated pressure field is measured in the focal plane $(x, y)$ and the axial plane $(x, z)$ and then compared to the theoretical field (see Fig. 6). For the selected spherical vortex of charge $m^{\prime}=1$, radial order $l=8$ and aperture angle $\alpha_{0}=49^{\circ}$, it appears from the figure that the high-amplitude ring is well recovered with good uniformity of amplitude. Inside the ring, the field is at zero amplitude and again is in agreement with the target theoretical one. Even though the variation of phase inside the small circle in the center is smeared out in the measured field, the global variation of the phase is in good agreement with the theoretical one. The modulus in axial plane $(x, z)$ is similar to the theory as expected. 

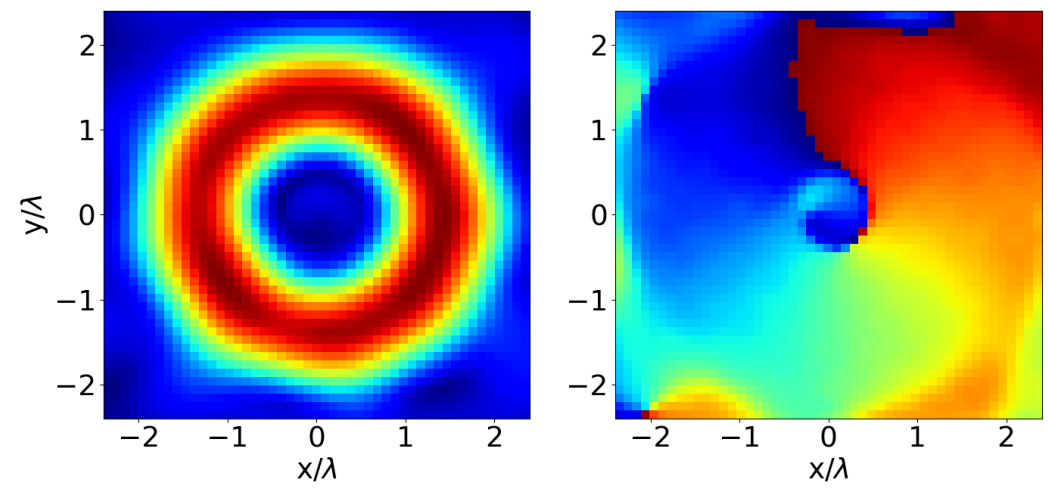

(a) Modulus and phase of the complex pressure field in plane $(x, y)$ (experimental measurements).
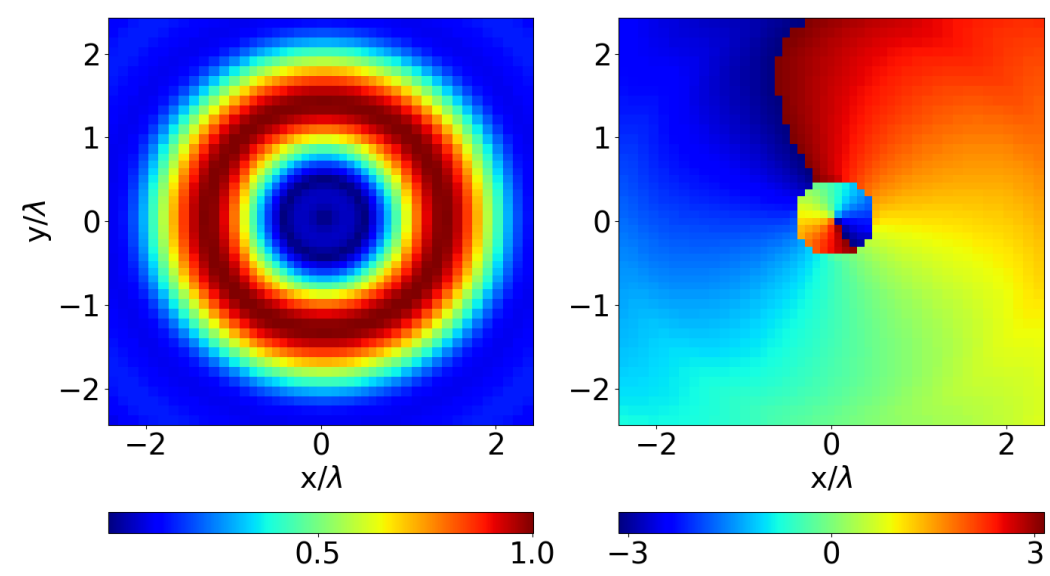

(b) Modulus and phase of the complex pressure field in plane $(x, y)$ (theoretical pressure field).
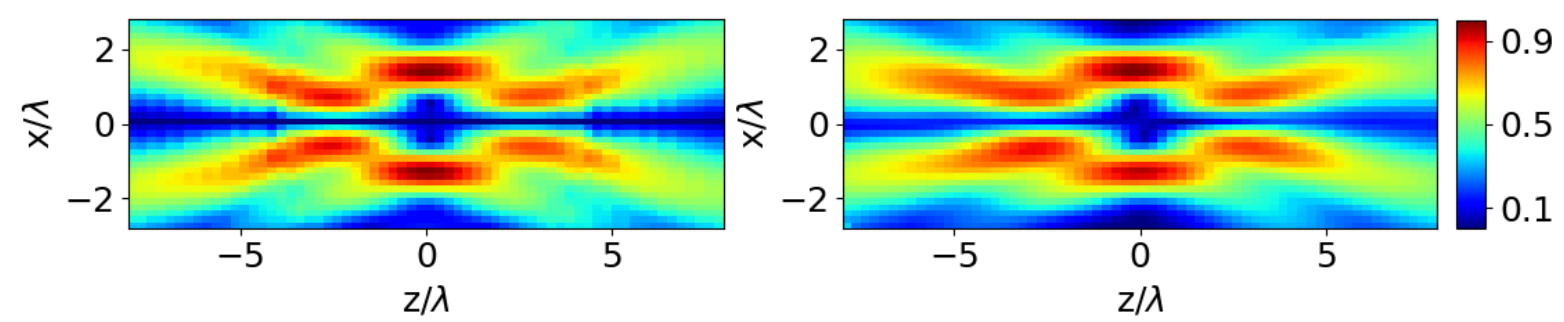

(c) Modulus of the complex pressure field in plane $(x, z)$ : theoretical (left) and experimental (right).

Figure 6: (Color online) Experimental and theoretical modulus and phase of the complex pressure field in plane $(x, y)$ and $(x, z)$ for a spherical vortex beam with $m^{\prime}=1, l=8$ and $\alpha_{0}=49^{\circ}$

Furthermore, from the measurement of the field in focal plane $(x, y)$, we can also compute the BSC and thus obtain the radiation forces by the angular spectrum method [44]. The radiation forces computed from the measurements are given in Fig. 7. We find a good agreement with the theoretical forces, 
especially for the axial force $F_{z}$ for which the discrepancy is less than $10 \%$. The latter confirms the high quality of the synthesis of spherical vortex beams for this distribution of control points.
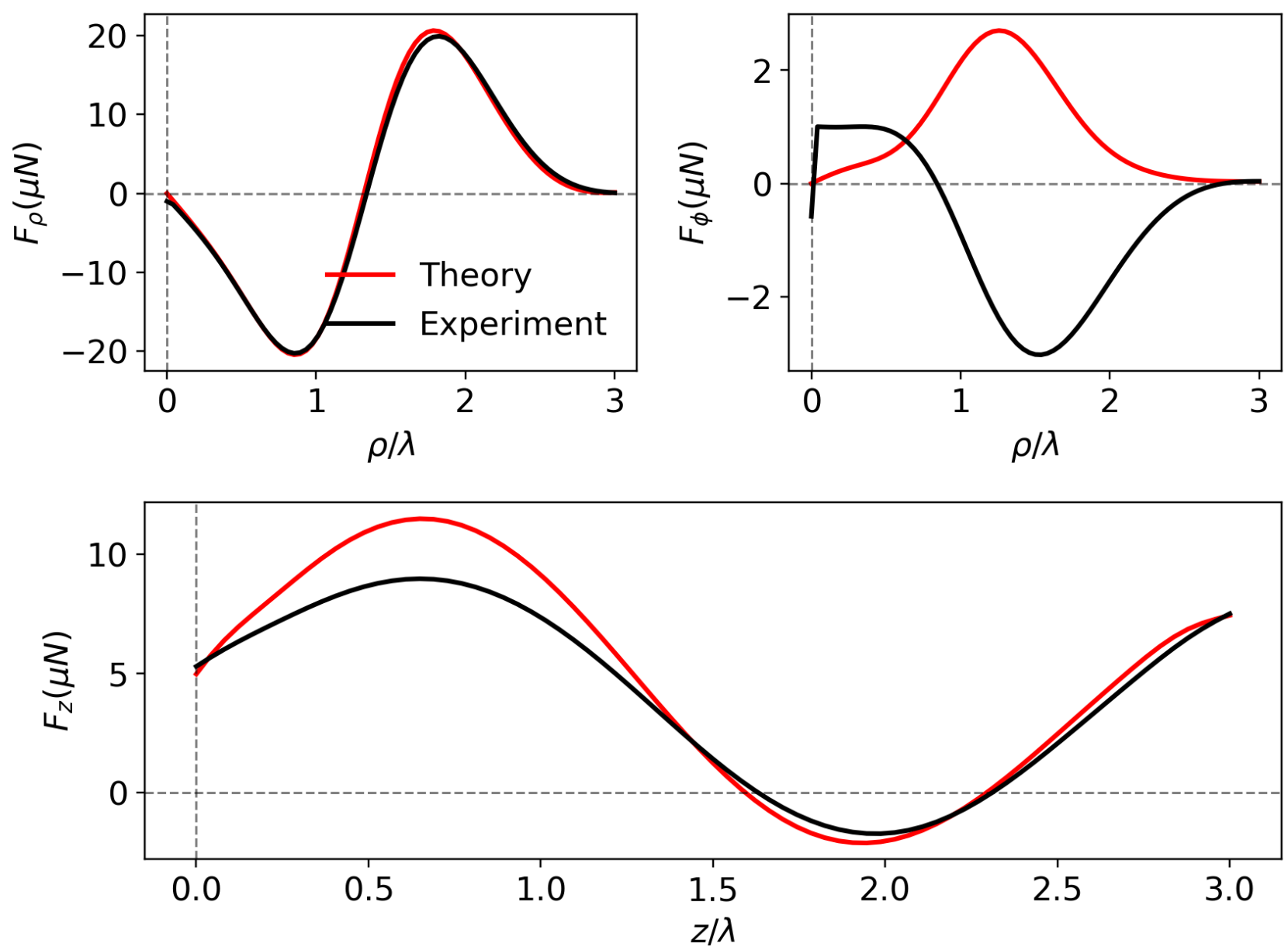

Figure 7: (Color online) Theoretical and experimentally estimated radiation force exerted on a polyethylene sphere of radius $a=0.45 \lambda$ by a focused spherical vortex beam of topological charge $m^{\prime}=1, l=8$.

Nevertheless, regarding the emission vectors obtained by the standard inverse filter method, the energy is mainly distributed on the first 20 transducers located in the middle and the other 100 transducers have barely no contribution to the pressure field. Each electronic channel can deliver high power, $25 \mathrm{~W}$, but for pulse signals only. Experiments of trapping and manipulation require tens of seconds of duration and hence the emitted power must be decreased. Taking into account the weight of our large targeted spheres, the applied axial force has to be as large as possible. For this reason, the emission vector is modified to increase the axial force in order to make optimal use of all the transducers and, at the same 
time, increase the amplitude of the high-amplitude ring.

Thus the following modifications are made: first of all, for each transducer, the emitted signals are normalized $e_{i}\left(t_{n}\right) / \max _{n}\left(e_{i}\left(t_{n}\right)\right)$, where $i$ is the index of the transducer (from 1 to 127) and $n$ is the index of the time sample. Then, each signal is scaled by a new amplitude factor equal to:

$$
\left(\max _{n}\left(e_{i}\left(t_{n}\right)\right) / \max _{i}\left(\max _{n}\left(e_{i}\left(t_{n}\right)\right)\right)\right)^{\beta}
$$

with $\beta$ equal to $1,0.5$ or 0.25 . By applying these modifications, the number of signals with significant amplitude increases. Meanwhile, the pressure field radiated and measured is also changed. In Fig. 8, the top row is the field measured with the original emission vector, $\beta=1$ and then going downward $\beta=0.5$ and finally $\beta=0.25$. In plane $(x, y)$, the high-amplitude rings keep the same pattern. While inside the rings, the pressure modulus is no longer zero and the phases have also been modified. Moreover, the differences between the pressure field measurements in plane $(x, z)$ are also clearly visible. 

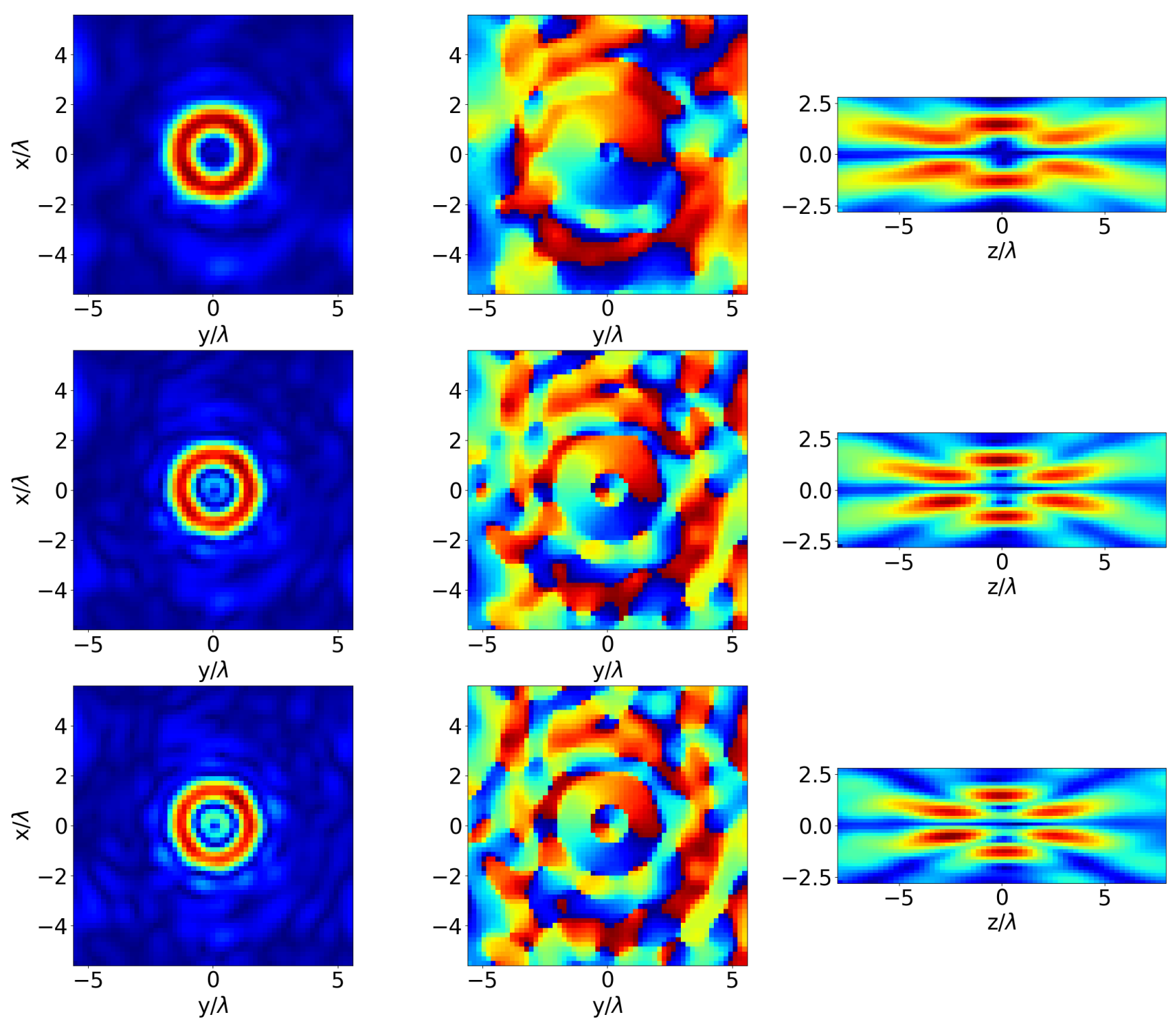

Figure 8: (Color online) Modulus (Left) and phase (Middle) of the complex pressure field in plane $(x, y)$ with modulus (Right) of the pressure field in plane $(x, z)$ for a spherical vortex beam with $l=8, \alpha_{0}=49^{\circ}$. From top to bottom: $\beta=1,0.5,0.25$. 

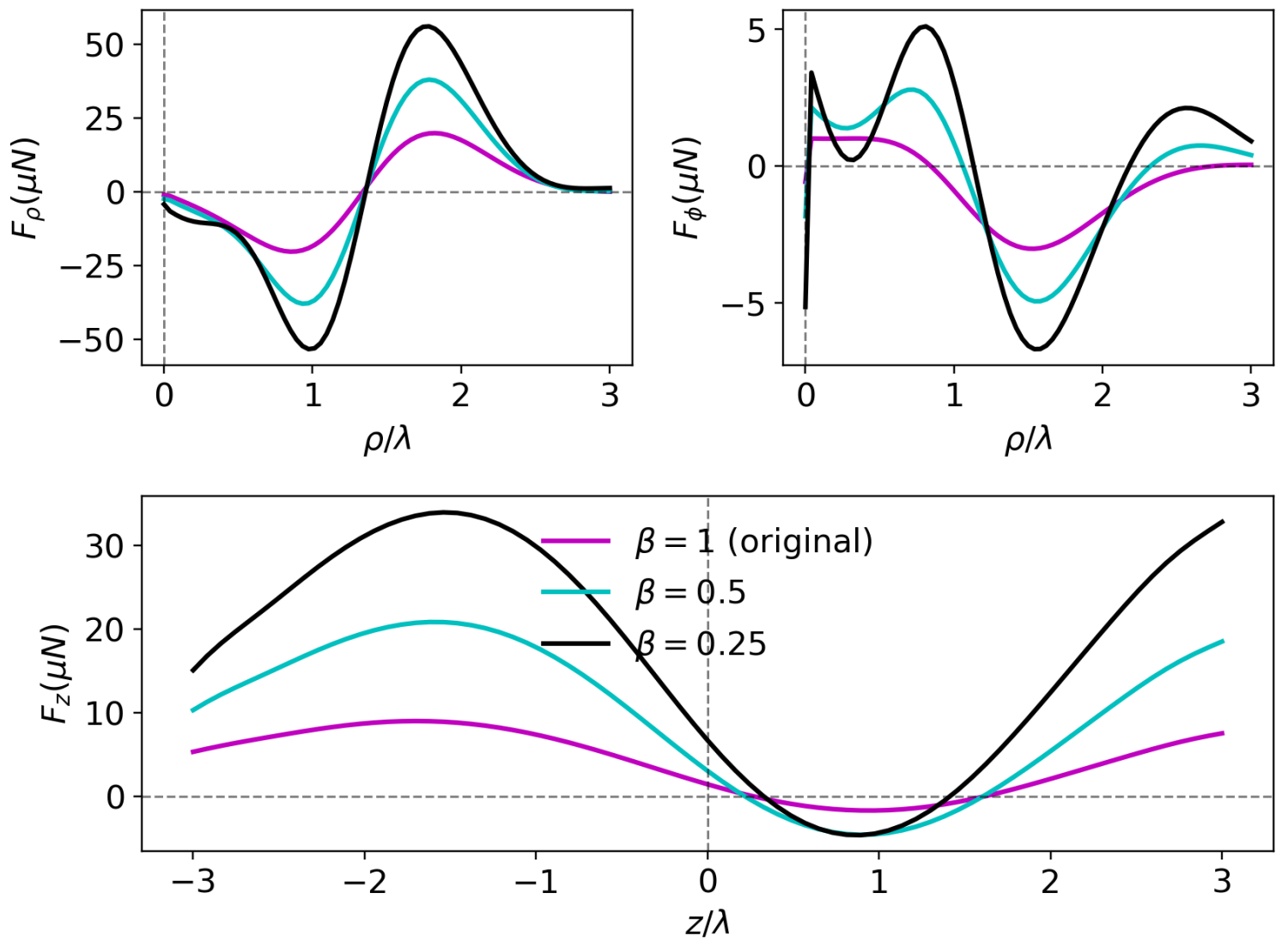

Figure 9: (Color online) Experimentally estimated radiation force exerted on a polyethylene sphere of radius $a=0.45 \lambda$ by a focused spherical vortex beam of topological charge $m^{\prime}=1, l=8$ synthesized by different emission vectors with $\beta=1$ (original), $0.5,0.25$.

In order to evaluate the impact of these modifications on the radiation forces quantitatively, we use again a calculation method based on the angular spectrum method which allows to estimate the radiation forces from the measured pressure fields [44]. As depicted in Fig. 9, by modifying the emission vector $\underline{E}$ from the origin up to $\beta=0.25$, the amplitude of the radial force increases and the trap in radial direction remains always. For the axial force, when $z<0$, the force is positive and increases notably with the decrease of the coefficient $\beta$. The maximum positive force with $\beta=0.25$ is four times larger than the original one. However, when the sphere is located behind the focus $(z>0)$, the maximum amplitude of the restoring force increases until $\beta=0.25$ and then decreases. Thus, according to the trapping performance illustrated by the radiation forces, the exponent $\beta=0.25$ is optimum and should be sufficient to overcome the weight of light spheres. This modification is used in the trapping experiments 
presented in the next section.

\subsection{Experimental trapping with a spherical vortex}

\subsubsection{Localization of the trapping zone}

One feature of the single side acoustical tweezers is to have a tight trapping zone. It means that the object can be trapped only in a very small region of the space, which is a very appealing property ensuring a very good selectivity of the device. Therefore, prior to any manipulation task, the trapping zone has to be found accurately. This is done with the hydrophone. The pressure field of the vortex in transverse plane $(x, y)$ has a zero amplitude zone at the center surrounded by a high-amplitude ring as shown in Fig. 1(b). At the focal plane, the amplitude of the ring reaches its maximum amplitude. The focal position of the vortex can be found by these characteristics. Once the hydrophone is successfully positioned at the focus, we then adjust the position of the camera to get a clear picture of the hydrophone, and at the same time make sure that the hydrophone is at the center of the image. This step of fixing the focal position allows later to locate the sphere at the trapping area of the vortex.

\subsubsection{Axial and radial traps}

Once the trapping zone is located by the hydrophone, the target beads are placed on the acoustically transparent membrane. By moving the membrane with the motorized positioning system, it is easy to move the target bead in the trapping zone of the spherical vortex beam. Once the bead is positioned inside the trapping zone, the spherical vortex is emitted to trap it. It should be noticed that since two types of polyethylene beads with different densities are used, two experimental setups are used. To trap the beads with density higher than water, the experimental setup used is the same as in Fig. 5. However, for the beads which float, the transducer array is placed under the membrane. During the experiments, once the field is activated, the membrane is moved transversely and axially. Particles could be selected individually and relatively to the membrane moved transversely. Fig. 10 shows three snapshots of such an experiment, see also video of the bead trapped and moved by the acoustic tweezers. We can see that the selected bead located in the center is radially trapped. Indeed while moving the polyethylene film, this particle doesn't move with the film and stays in the same position while other beads located in the surrounding and not trapped are moving with the membrane and appear in the camera field of view. On the contrary, no axial trap was observed during the experiments: when the membrane is moved in the 
direction of propagation of the waves, the bead also moves. The reasons responsible for the failure of the axial trap have caught our attention and are discussed in the following sections.

Figure 10: Snapshots of a polyethylene sphere trapped radially by a spherical vortex. A movie is available on line showing the bead trapped and moved laterally by the tweezers.

\section{Discussion and conclusions}

\subsection{The resonance of the spheres}

Despite the theoretical predictions and the good wavefield synthesis, only the radial trap has been observed and no evidence of the axial trap have been found. Even though the emission vectors have been modified to ensure a negative axial force larger than the sphere weight, this was not strong enough to take off the sphere. In the following section, we revisit the numerical evaluation in order to detail the choice of parameters (material, size) selected to ensure an efficient trapping. According to our estimation of the axial forces for a polyethylene sphere of different radii, the trapping capability of the spherical vortex, especially the axial trap, is not constant. As depicted in Fig. 11, among the radii tested for the polyethylene spheres $a=0.41 \lambda, a=0.45 \lambda$ and $a=0.48 \lambda$, the radial trap exists always. On the contrary, only the sphere with $a=0.45 \lambda$ can be trapped axially. 

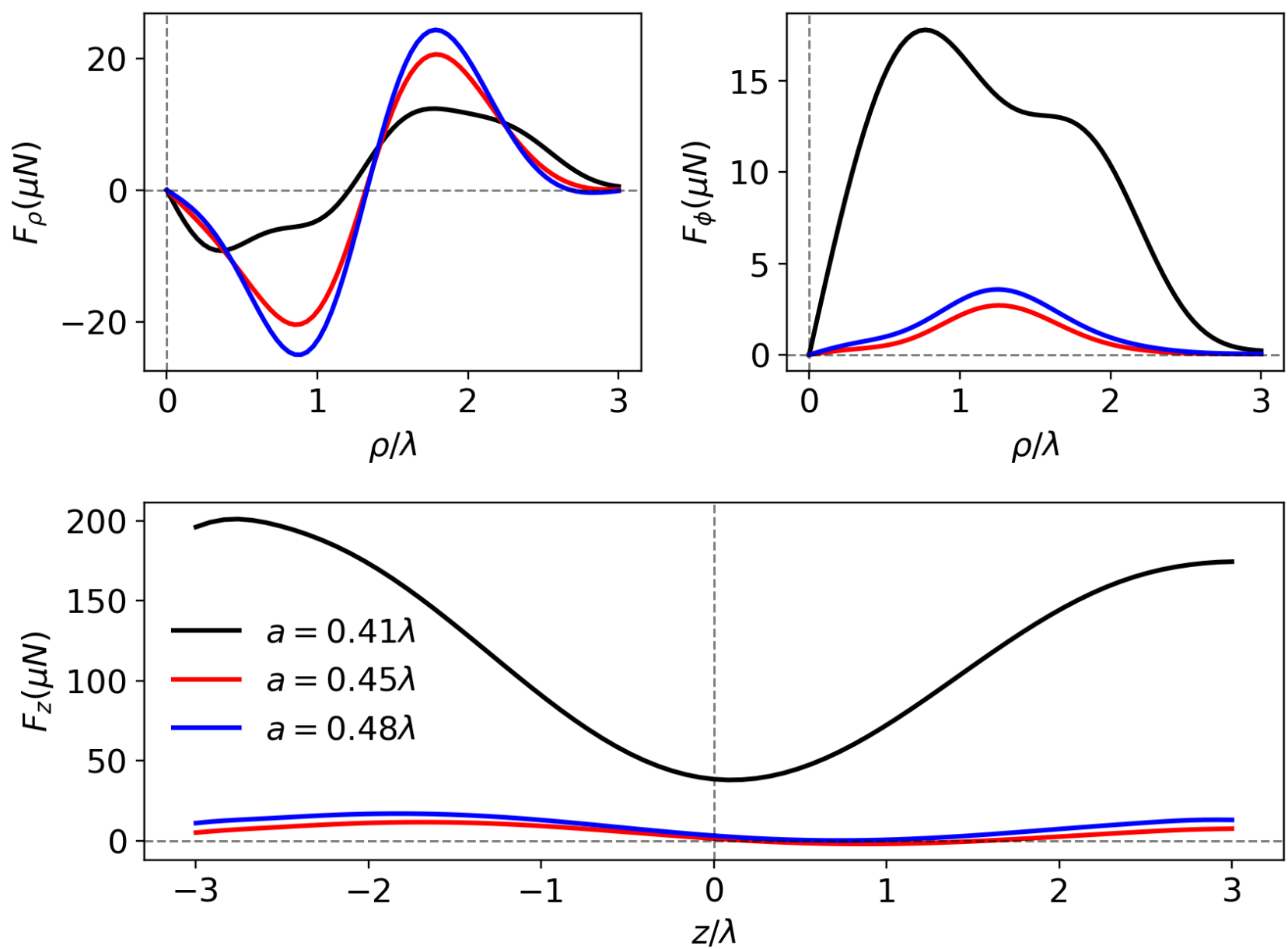

Figure 11: (Color online) Radial $F_{\rho}$, azimuthal $F_{\phi}$, axial $F_{z}$ forces exerted on a polyethylene sphere of radii $a=0.41 \lambda$, $a=0.45 \lambda, a=0.48 \lambda$ by a spherical vortex beam.

Therefore, to succeed in trapping the particles experimentally, it is important to test the range of sizes and materials that can be trapped, especially in the axial direction of a spherical vortex. The negative force in the axial direction is the key to trap the particles. Thus, the determination of the suitable range of sphere sizes requires only to compute the axial force at a position where the negative force is most likely to appear. This kind of analysis on negative axial force was first demonstrated for a Bessel beam [27]. Note that the maximum axial restoring force exerted by a spherical vortex at frequency $1 \mathrm{MHz}$ is about $5 \mu \mathrm{N}$ (see Fig. 11), however the volume of the sphere is about $1 \mathrm{~mm}^{3}$ which limits the choice of sphere materials to light polymer spheres with density comparable to water. Therefore, only light spheres like polyethylene and polystyrene are analyzed in this section. According to Fig. 11, for instance, at $z=0.9 \lambda$ 
the axial force is negative with a maximum amplitude for a polyethylene sphere of $a=0.45 \lambda$. In Fig. $12(\mathrm{a})$, the axial force at $z=0.9 \lambda$ as a function of sphere radius is represented. As illustrated in the figure, from $a=0.2 \lambda$, the resonances begin to appear in the axial force and thereafter regions for which the axial force is negative alternates with regions where the force is positive. In the figure, the zone where $F_{z}<0$ represents the range of sphere radius that can be trapped. Only the spheres with radii between $(0.42 \lambda$ and $0.47 \lambda)$ or $(0.5 \lambda$ and $0.54 \lambda),(0.59 \lambda$ and $0.64 \lambda)$ have a negative force and therefore can be trapped by the spherical vortex. This result agrees with the force estimation in Fig.11, since the spheres of radii $a=0.41 \lambda$ and $a=0.48 \lambda$ are at the resonances of the axial force, it is impossible to trap them axially. These resonances of the force come from the resonances of the scattering coefficients $R_{n}$. The expression of the axial force [6] can be calculated with :

$$
F_{z}=-2 \frac{\langle V\rangle}{k_{0}^{2}} \sum_{n=0}^{\infty} \sum_{|m| \leq n} \Im\left(G_{n}^{m} A_{n}^{m *} A_{n+1}^{m} C_{n}\right) .
$$

With:

$$
\begin{aligned}
& V=p_{a}^{2} /\left(4 \rho_{0} c_{0}^{2}\right), \\
& C_{n}=R_{n}^{*}+R_{n+1}+2 R_{n}^{*} R_{n+1}, \\
& G_{n}^{m}=\sqrt{(n+m+1)(n-m+1)} / \sqrt{(2 n+1)(2 n+3)} .
\end{aligned}
$$

where $A_{n}^{m}$ are the BSC defined by Eq. 3, $C_{n}$ is a function of the scattering coefficients $R_{n} ; p_{a}$ is the linear component of the acoustic pressure; $\rho_{0}$ and $c_{0}$ are the fluid density and speed of sound at rest respectively. In Fig. 12(b), the modulus of $C_{n}$ is plotted for a polyethylene sphere as a function of radius. Comparing Fig. 12(a) and Fig. 12(b), the resonances in $C_{n}$ locate at the same positions as the resonances observed in the axial forces. The figure also illustrates that for $a<0.6 \lambda$, the coefficients $C_{n}$ with $n>6$ can be neglected since from $n=6$ the amplitudes become weak. As the force is a sum on degree $\mathrm{n}$ of function:

$$
\hat{f}_{z}^{n}=-\Im\left(G_{n}^{m} A_{n}^{m *} A_{n+1}^{m} C_{n}\right)
$$

The contribution of $\hat{f}_{z}^{n}$ to the negative force at each degree $\mathrm{n}$ can be computed by Eq. (8). In Fig. $12(\mathrm{c})$, the $\hat{f}_{z}^{n}$ until degree $n=6$ are depicted. Taking the trapping region between $0.42 \lambda$ and $0.47 \lambda$ as an example, according to the figure, $\hat{f}_{z}^{n}$ with negative amplitudes that contribute to the final negative force 
are at degrees $n=2, n=3$ and $n=4$.

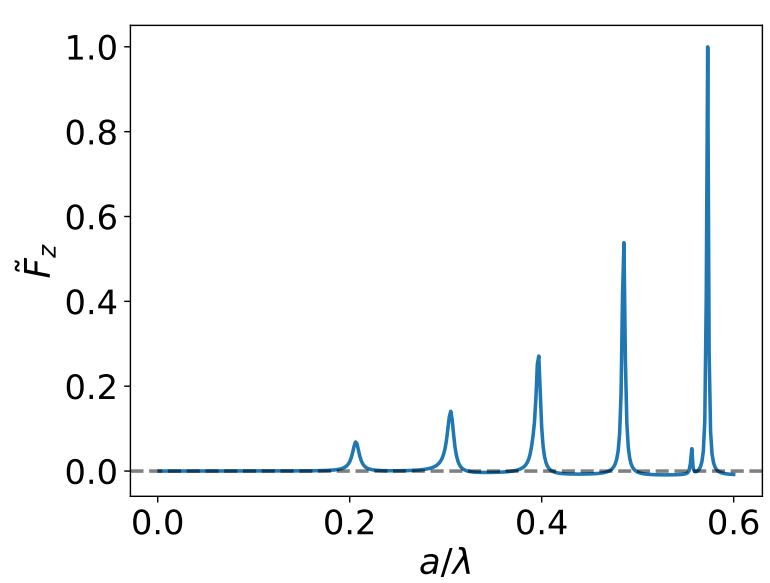

(a) $F_{z}$.

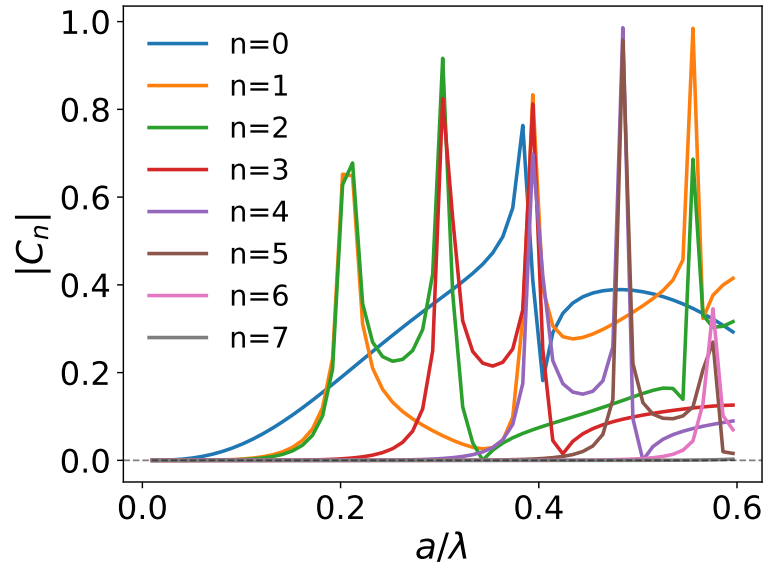

(b) $C_{n}$.

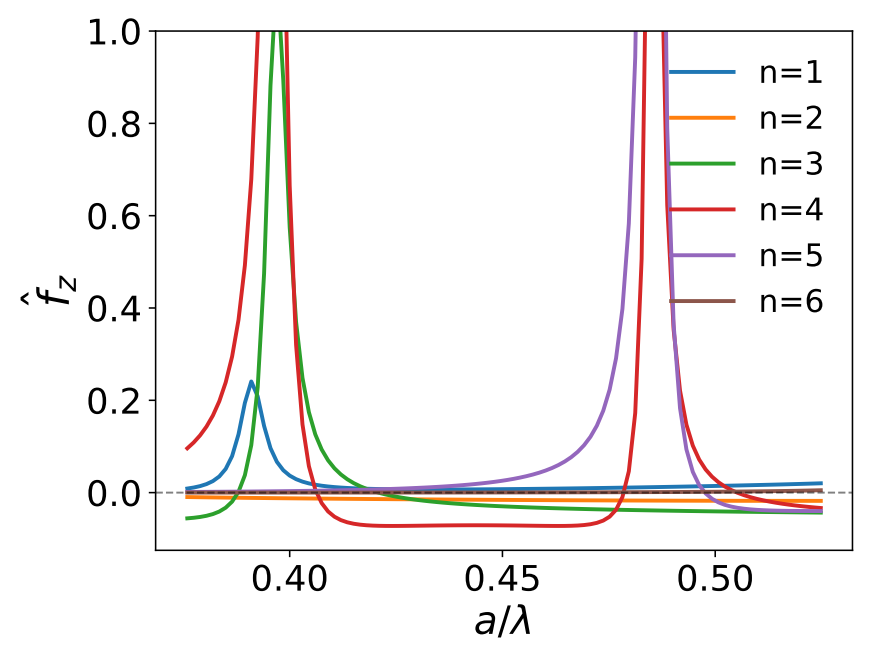

(c) $\hat{f}_{z}^{n}$

Figure 12: (Color online) (a) Normalized axial forces $\tilde{F}_{z}$ exerted on a polyethylene sphere positioned at $z=0.9 \lambda$ as a function of sphere radius by a spherical vortex ; (b) Modulus of $C_{n}$ as a function of sphere radius for a polyethylene sphere; (c) $\hat{f}_{z}^{n}$ as a function of sphere radius for different degrees until $n=6$ for a polyethylene sphere.

In order to find the relation between the coefficients $C_{n}$ and its contribution to the negative force, the

coefficients $C_{n}$ of polyethylene sphere at degree $n=2, n=3$ and $n=4$ are depicted in Fig. 13(a) and 13(b). In the figure, the region within which the negative force appears is highlighted by two gray dashed curves. In the trapping region, the modulus of $C_{n}$ as well as the phase are constant and located between two resonances. The phase inside the trapping region is approximately $\frac{\pi}{2}$. As the axial force component 


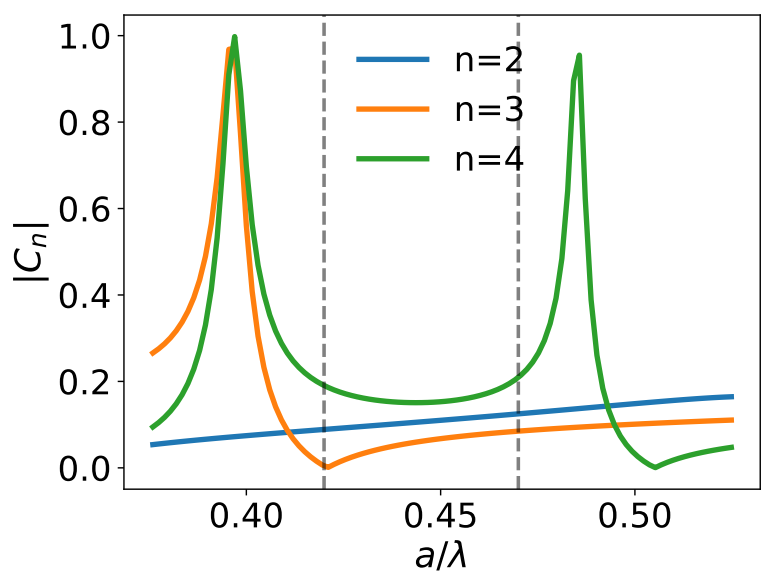

(a) Modulus of $C_{n}$.

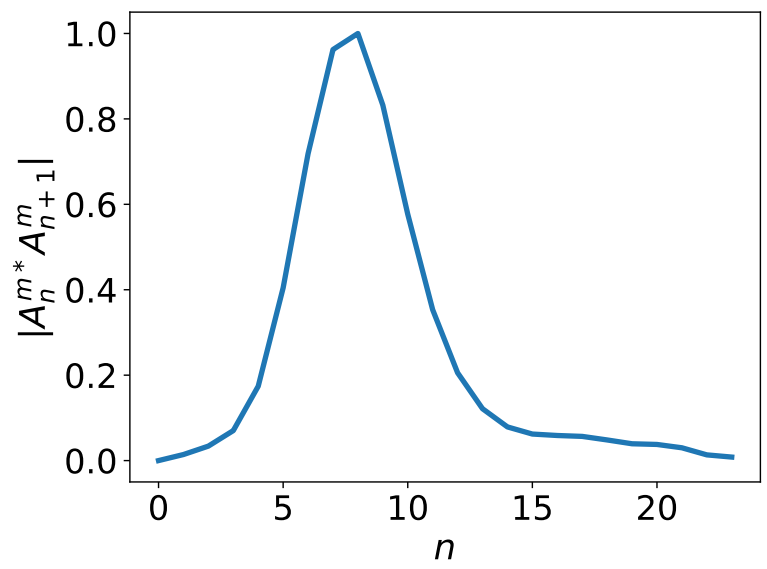

(c) Modulus of $A_{n}^{m *} A_{n+1}^{m}$

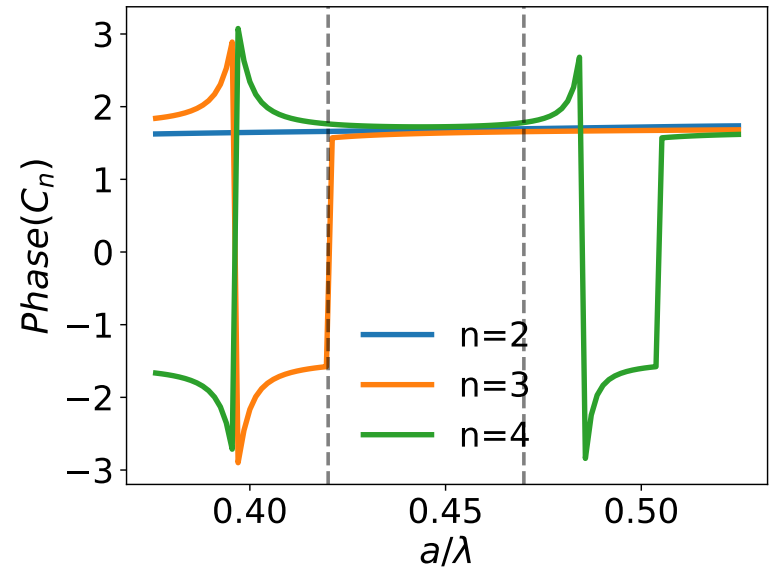

(b) Phase of $C_{n}$

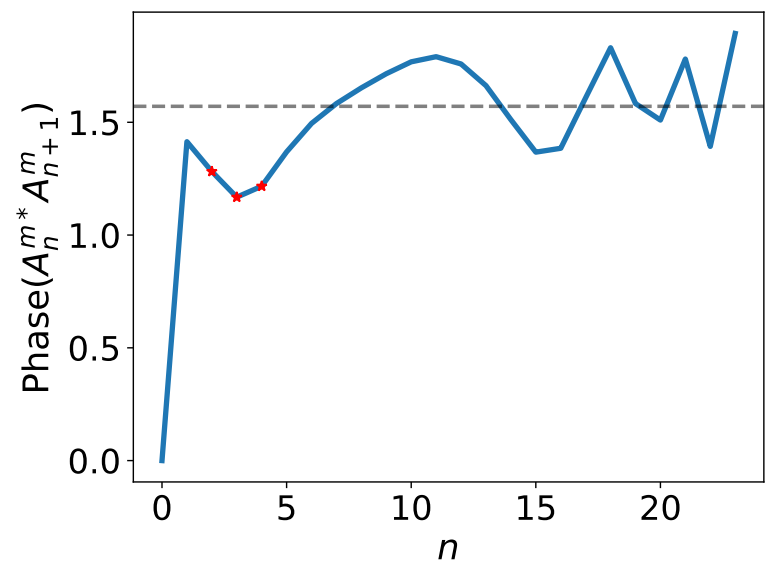

(d) Phase of $A_{n}^{m *} A_{n+1}^{m}$

Figure 13: (Color online) (a) Modulus and (b) phase of $C_{n}$ as a function of radius at degrees $n=2, n=3, n=4$ for a polyethylene sphere; (c) Modulus and (d) phase of $A_{n}^{m *} A_{n+1}^{m}$ as a function of degree $\mathrm{n}$.

for each $n$ is the opposite of the imaginary part of the product of $A_{n}^{m *} A_{n+1}^{m}$ and $C_{n}$, the axial force is negative when the phase is inferior to $\pi$. In Fig. 13(c) and 13(d), the modulus and phase of $A_{n}^{m *} A_{n+1}^{m}$ are plotted. Even though the maximum of the modulus is at $n=8$, the corresponding phase is however above $\frac{\pi}{2}$ (the gray dashed line represents the position where the phase is $\pi / 2$ ), since the phase of $C_{n}$ is equal to $\frac{\pi}{2}$, the phase of the product is then equal or superior to $\pi$ and does not contribute to the axial restoring force at all (for a value of $\pi$ ) and will, on the contrary, push away the sphere for values superior to $\pi$. Meanwhile, at degree $n=2, n=3$ and $n=4$, the phases of $A_{n}^{m *} A_{n+1}^{m}$ are the smallest and located below $\frac{\pi}{2}$ which finally contributes to the negative restoring forces. 
The trap capability of the spherical vortex on a polystyrene sphere can be examined in the same way. The axial force exerted on a polystyrene sphere at position $z=0.9 \lambda$ as a function of the polystyrene sphere radius is computed and shown in Fig. 14(a). According to the figure, the negative force appears at some of the radii but the amplitude is very weak. For one of the regions between $a=0.46 \lambda$ and $0.49 \lambda$, the $\hat{f}_{z}^{n}$ are plotted in Fig. $14(\mathrm{~b})$. The $\hat{f}_{z}^{n}$ contributing to the negative force are at degrees $n=2$, $n=3, n=4$ again. Then we plot the coefficients $C_{n}$ as shown in Fig. 14(c) and 14(d). Similar to the polyethylene sphere, inside the trapping zone, the modulus and phase of $C_{n}$ are constant. The phases at degree $n=2$, and $n=3$ are close to $\frac{\pi}{2}$ while at degree $n=4$, the phase is larger and is equal to $\frac{2 \pi}{3}$. As 270 the sum of $C_{n}$ phase and $A_{n}^{m *} A_{n+1}^{m}$ phase at $n=4$ is close but less than $\pi$ this results in a small negative force. Moreover, since the sinus function (the imaginary part) decreases linearly around $\pi$, a decrease of phase thus results in a proportional increase of the modulus of the negative force. 


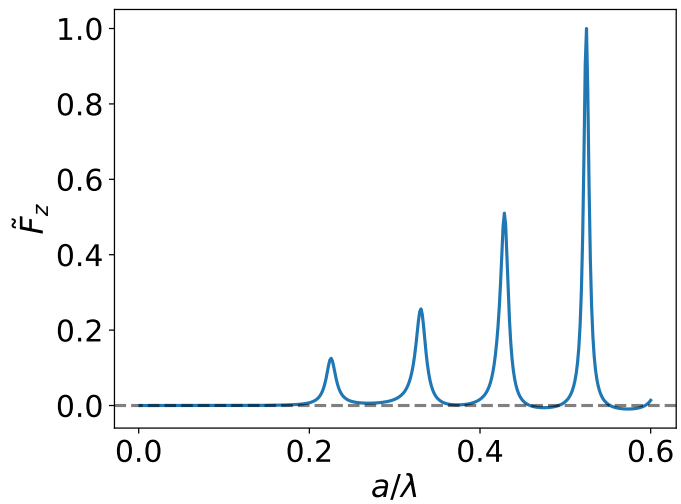

(a) Normalized axial $F_{z}$ forces

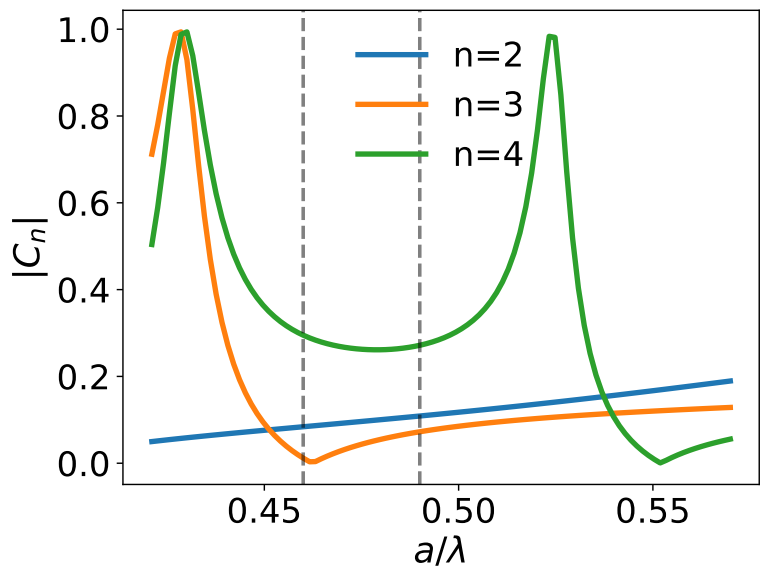

(c) Modulus of $C_{n}$

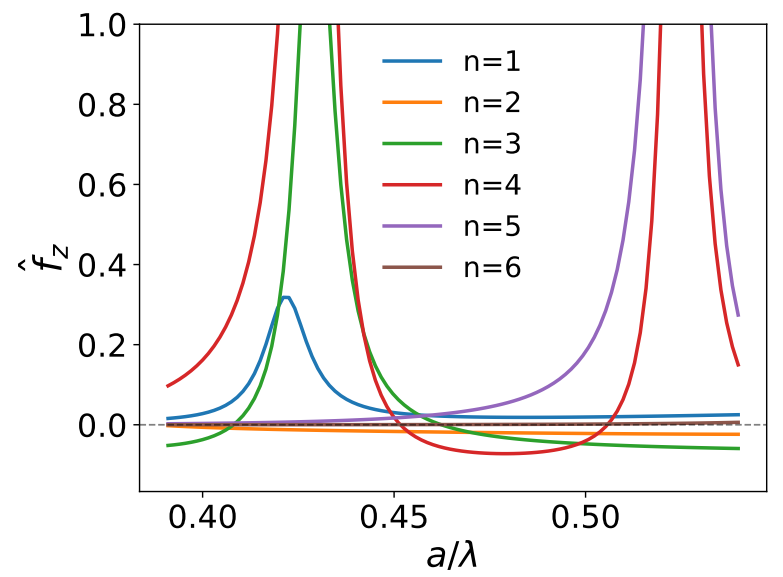

(b) $\hat{f}_{z}^{n}$

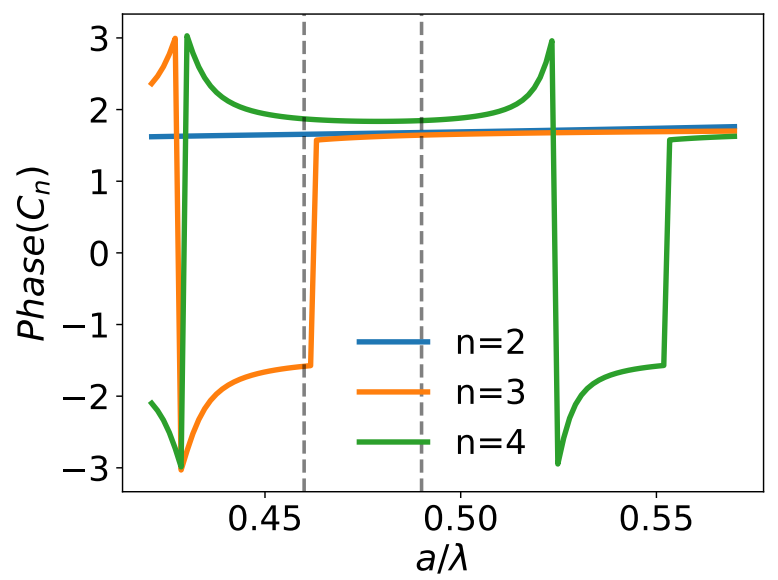

(d) Phase of $C_{n}$

Figure 14: (Color online) (a) Normalized axial $F_{z}$ forces exerted on an polystyrene sphere positioned at $z=0.9 \lambda$ as a function of sphere radius; (b) $\hat{f}_{z}^{n}$ as a function of sphere radius for different degrees until $n=6$; (c) Modulus and (d) phase of $C_{n}$ as a function of sphere radius at degrees $n=2, n=3, n=4$ for a polystyrene sphere.

\subsection{Influence of the BSC}

As the scattering coefficients $C_{n}$ of a polystyrene sphere depends only on its physical properties, the only way to trap a polystyrene sphere is to adjust the BSC of the spherical vortex beam to give rise to the negative force. The BSC of a spherical vortex, as demonstrated in the previous section, can be modified by changing the aperture angle $\alpha_{0}$, the radial degree $l$, or topological charge $m^{\prime}$. Taking the radial degree $l$ as an example, in Fig. 15(a) and 15(b), the modulus and phase of $A_{n}^{m *} A_{n+1}^{m}$ with different radial degrees are shown. The gray dashed lines highlight the radial degrees of interest. Looking at the phases of 
each radial degree, the $l=8$ gives rise to a phase significantly below $\pi / 2$ in the region of interest and hence potentially to a large negative force. However, the modulus of the $A_{n}^{m *} A_{n+1}^{m}$, at the same degree $n$, decrease when $l$ increases and this trend is in favor of the smallest $l$. Therefore the impact of changing the radial degree $l$ on the negative force is not obvious. In Fig. $15(\mathrm{c}), \hat{f}_{z}^{n}$ as a function of polystyrene sphere radius with different radial degrees $l$ selected to compute the the BSC are shown. From the figure, the BSC with radial degree $l=8$ lead to the most significant negative force. In spite of the fact that the modulus of $A_{n}^{m *} A_{n+1}^{m}$ at $n=2,3,4$ with $l=8$ are smaller than those computed with $l=6$, and $l=7$, they have a smaller phase at the same time. Thus again, this result emphasizes that the phase of the scattered waves relative to the incident one is the main feature leading to a large enough negative restoring force and hence a $3 \mathrm{D}$ trapping. 


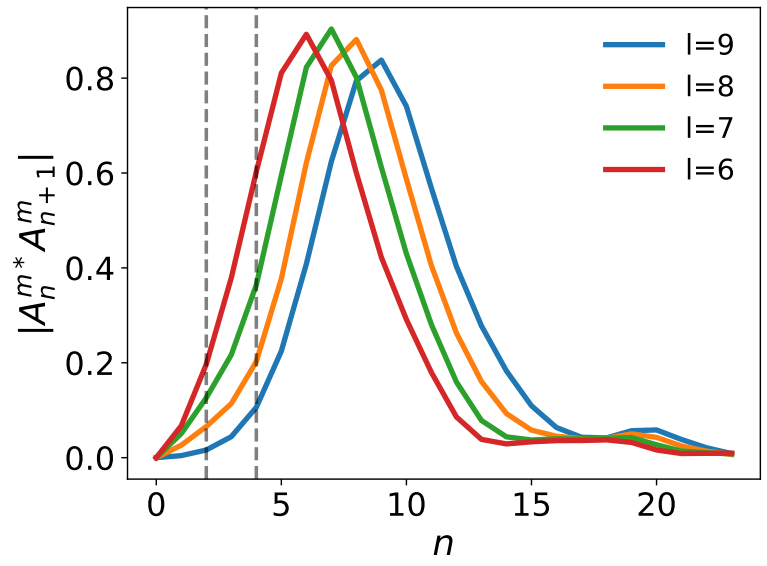

(a) Modulus of $A_{n}^{m *} A_{n+1}^{m}$

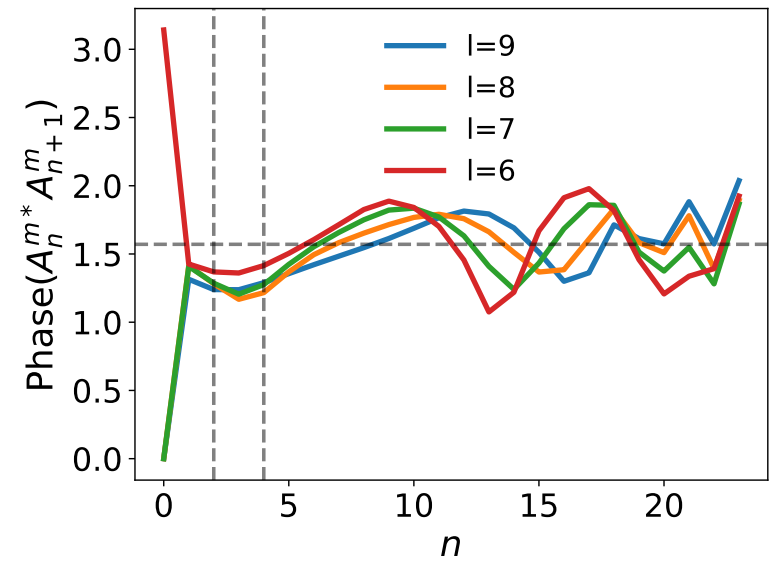

(b) Phase of $A_{n}^{m *} A_{n+1}^{m}$

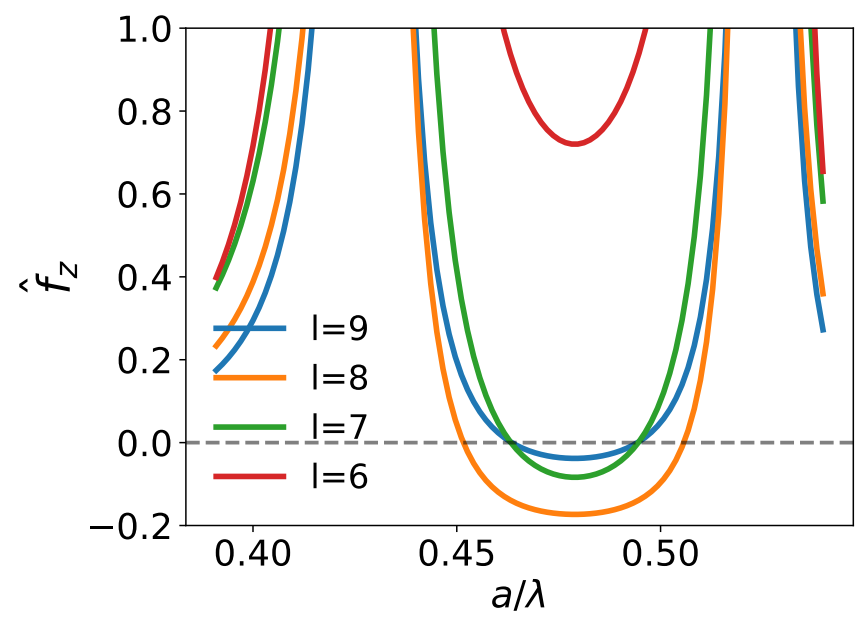

(c) $\hat{f}_{z}^{n}$

Figure 15: (Color online) (a) Modulus and (b) phase of $A_{n}^{m *} A_{n+1}^{m}$ as a function of degree $n$ for different radial degrees $l$; (c) $\hat{f}_{z}^{n}$ at degree $n=4$ as a function of sphere radius for different radial degrees $l$.

\subsection{Conclusions}

In conclusion, diffraction in the far-field limits the focal spot to a size larger than about one wavelength, therefore achieving acoustical tweezers with optimal selectivity means trapping object of about the size of the tweezers and hence of about one wavelength also. However for such large objects, the modes have resonances due to creeping waves or anti-resonances around which the phase and the amplitude of the modal reflection coefficient vary rapidly. Since the trapping ability of a spherical vortex is strongly dependent on the phase of the scattered wave relative to the incident one, this effect leads to 
a reversal of the radiation force direction. Different kinds of acoustical vortices were assessed. As anticipated, the so-called spherical vortices with their peculiar energy density that seems to bypass a zone where ultrasound is canceled give the best results. The sharp energy density gradient in both radial and axial directions are favorable for a strong restoring force in all directions. With such fields, we predict that $3 \mathrm{D}$ trapping can be achieved between two resonance peaks for spheres of about one wavelength in diameter and with an axial force strong enough to exceed the sphere weight. While we observed radial trapping, the axial force obtained experimentally was not sufficient to lift the sphere. Several explanations are possible for this partial failure. First the physical properties of the polymer sphere are not well characterized. A change of the speed of sound for either shear or longitudinal waves will result in a variation of the speed of sound of the creeping waves and hence a shift in frequency of the resonances. Moreover, these polymers are visco-elastic and the acoustic attenuation will contribute to pushing the sphere and hence the axial restoring force is decreased. Since this parameter is not known, we did not try to estimate the amplitude of this effect. Second, there are electrical static forces between polyethylene spheres and a surfactant must be used to disperse them in water. Since our membrane is also in polyethylene, we did observe that a static force occurs between the spheres and the membrane. The amplitude of this static force is difficult to estimate and to mitigate but has to be overcome by the axial radiation force to take off the sphere. So, it may be possible that the force is enough to compensate the weight but too small to compensate the weight and overcome the short ranged static force that exists when the sphere lies on the membrane. In this study, the relatively low frequency and hence relatively large wavelength leads to polymer materials for which the density contrast with the fluid is weak to mitigate the weight of the object. Such constraint will not exist if we scale down the experimental conditions. Indeed, the sphere weight is proportional to its volume and hence decreases with the cube of the wavelength and hence faster than the radiation force amplitude. Denser materials are generally much more stiffer than polymers and therefore the speed of sound is higher. Such conditions are favorable since the resonances will be shifted to a higher ratio between the radius and the wavelength in water. Moreover material like glass are well characterized, the attenuation can be neglected and no electrical force with the membrane is expected. A numerical method efficient enough to search for the parameters (numerical aperture, elastic constants, frequency...) allowing to obtain a trap in the three directions must be developed to carry out this task. 


\section{Acknowledgments}

This work was supported by French state funds managed by the ANR within the Programme Investissements d'Avenir under reference ANR-11-IDEX-0004-02, and more specifically within the framework of the Cluster of Excellence MATISSE led by Sorbonne Universités.

\section{References}

[1] Arai, F., Ng, C., Maruyama, H., Ichikawa, A., El-Shimy, H., Fukuda, T., 2005. On chip single-cell separation and immobilization using optical tweezers and thermosensitive hydrogel. Lab Chip 5, $1399-1403$.

[2] Arlt, J., Padgett, M.J., 2000. Generation of a beam with a dark focus surrounded by regions of higher intensity: the optical bottle beam. Opt. Lett. 25, 191-193.

[3] Ashkin, A., 1992. Forces of a single-beam gradient laser trap on a dielectric sphere in the ray optics regime. Biophys. J. 61, 569-582.

[4] Ashkin, A., Dziedzic, J.M., Bjorkholm, J., Chu, S., 1986. Observation of a single-beam gradient force optical trap for dielectric particles. Opt Lett 11, 288-290.

[5] Baresch, D., Thomas, J.L., Marchiano, R., 2013a. Spherical vortex beams of high radial degree for enhanced single-beam tweezers. J. Appl. Phys. 113, 184901.

[6] Baresch, D., Thomas, J.L., Marchiano, R., 2013b. Three-dimensional acoustic radiation force on an arbitrarily located elastic sphere. J. Acoust. Soc. Am. 133, 25-36.

[7] Baresch, D., Thomas, J.L., Marchiano, R., 2016. Observation of a single-beam gradient force acoustical trap for elastic particles: acoustical tweezers. Phys. Rev. Lett. 116, 024301.

[8] Baresch, D., Thomas, J.L., Marchiano, R., 2018. Orbital angular momentum transfer to stably trapped elastic particles in acoustical vortex beams. Phys. Rev. lett. 121, 074301.

[9] Blasquez, A., 2019. Optical tweezers: Phototoxicity and thermal stress in cells and biomolecules. Micromachines 10, 507. 
[10] Bokor, N., Davidson, N., 2007. A three dimensional dark focal spot uniformly surrounded by light. Opt. Commun. 279, 229-234.

[11] Chen, X., Lam, K.H., Chen, R., Chen, Z., Yu, P., Chen, Z., Shung, K.K., Zhou, Q., 2017. An adjustable multi-scale single beam acoustic tweezers based on ultrahigh frequency ultrasonic transducer. Biotechnol. Bioeng. 114, 2637-2647.

[12] Choe, Y., Kim, J.W., Shung, K.K., Kim, E.S., 2011. Microparticle trapping in an ultrasonic bessel beam. Appl. phys. lett. 99, 233704.

[13] Courtney, C.R., Drinkwater, B.W., Demore, C.E., Cochran, S., Grinenko, A., Wilcox, P.D., 2013. Dexterous manipulation of microparticles using bessel-function acoustic pressure fields. Appl. Phys. Lett. 102, 123508.

[14] Fink, M., 1992. Time reversal of ultrasonic fields. i. basic principles. IEEE Trans Ultrason Ferroelectr Freq Control 39, 555-566.

[15] Hefner, B.T., Marston, P.L., 1999. An acoustical helicoidal wave transducer with applications for the alignment of ultrasonic and underwater systems. J. Acoust. Soc. Am. 106, 3313-3316.

[16] Jiménez, N., Picó, R., Sánchez-Morcillo, V., Romero-García, V., García-Raffi, L.M., Staliunas, K., 2016. Formation of high-order acoustic bessel beams by spiral diffraction gratings. Phys. Rev. E 94, 053004.

[17] Jin, Y., Kumar, R., Poncelet, O., Mondain-Monval, O., Brunet, T., 2019. Flat acoustics with soft gradient-index metasurfaces. Nat Commun 10, 143.

[18] Khonina, S.N., Golub, I., 2013. Engineering the smallest 3d symmetrical bright and dark focal spots. J. Opt. Soc. Am. A 30, 229-234.

[19] Lee, J., Teh, S.Y., Lee, A., Kim, H.H., Lee, C., Shung, K.K., 2009. Single beam acoustic trapping. Appl. phys. lett. 95, 073701.

[20] Lee, J., Teh, S.Y., Lee, A., Kim, H.H., Lee, C., Shung, K.K., 2010. Transverse acoustic trapping using a gaussian focused ultrasound. Ultrasound Med Biol 36, 350-355. 
[21] Lim, H.G., Kim, H.H., Yoon, C., Shung, K.K., 2019. A one-sided acoustic trap for cell immobilization using 30-MHz array transducer. IEEE Trans Ultrason Ferroelectr Freq Control 67, 167-172.

[22] Lirette, R., Mobley, J., Zhang, L., 2019. Ultrasonic extraction and manipulation of droplets from a liquid-liquid interface with near-field acoustic tweezers. Phys. Rev. Applied 12, 061001.

[23] Liu, Y., Cheng, D., Sonek, G., Berns, M., Chapman, C., Tromberg, B., 1995. Evidence for localized cell heating induced by infrared optical tweezers. Biophys. J. 68, 2137-2144.

[24] Mammen, M., Helmerson, K., Kishore, R., Choi, S.K., Phillips, W.D., Whitesides, G.M., 1996. Optically controlled collisions of biological objects to evaluate potent polyvalent inhibitors of viruscell adhesion. Chem. Biol. 3, 757-763.

[25] Marchiano, R., Thomas, J.L., 2005. Synthesis and analysis of linear and nonlinear acoustical vortices. Phys. Rev. E 71, 066616.

[26] Marston, P.L., 2006. Axial radiation force of a bessel beam on a sphere and direction reversal of the force. J. Acoust. Soc. Am. 120, 3518-3524.

[27] Marston, P.L., 2007. Negative axial radiation forces on solid spheres and shells in a bessel beam. J. Acoust. Soc. Am. 122, 3162-3165.

[28] Marzo, A., Seah, S.A., Drinkwater, B.W., Sahoo, D.R., Long, B., Subramanian, S., 2015. Holographic acoustic elements for manipulation of levitated objects. Nat Commun 6, 8661.

[29] Menon, R., Rogge, P., Tsai, H.Y., 2009. Design of diffractive lenses that generate optical nulls without phase singularities. J. Opt. Soc. Am. A 26, 297-304.

[30] Neuman, K.C., Nagy, A., 2008. Single-molecule force spectroscopy: optical tweezers, magnetic tweezers and atomic force microscopy. Nat. Methods 5, 491.

395 [31] Piché, L., 1984. Ultrasonic velocity measurement for the determination of density in polyethylene. Polymer Engineering \& Science 24, 1354-1358. 
[32] Piché, L., 1987. Application of ultrasonics to the characterization of composites: A method for the determination of polyethylene density, in: Bussière, J., Monchalin, J., Ruud, C., Green, R. (Eds.), Nondestructive characterization of materials II. Springer, Boston, MA, pp. 79-87.

[33] Riaud, A., Baudoin, M., Matar, O.B., Becerra, L., Thomas, J.L., 2017. Selective manipulation of microscopic particles with precursor swirling rayleigh waves. Phys. Rev. App. 7, 024007.

[34] Silva, G.T., Baggio, A.L., 2015. Designing single-beam multitrapping acoustical tweezers. Ultrasonics 56, 449-455.

[35] Skidanov, R., Kachalov, D., Khonina, S., Porfirev, A., Pavelyev, V., 2015. Three-dimensional laser trapping on the base of binary radial diffractive optical element. J. Mod. Opt. 62, 1183-1186.

[36] Svoboda, K., Block, S.M., 1994. Biological applications of optical forces. Annu. Rev. Biophys. Biomol. Struct. 23, 247-285.

[37] Tanter, M., Thomas, J.L., Fink, M., 2000. Time reversal and the inverse filter. J. Acoust. Soc. Am. $108,223-234$.

[38] Thomas, J.L., Marchiano, R., 2003. Pseudo angular momentum and topological charge conservation for nonlinear acoustical vortices. Phys. Rev. lett. 91, 244302.

[39] Trinh, E., 1985. Compact acoustic levitation device for studies in fluid dynamics and material science in the laboratory and microgravity. Rev Sci Instrum 56, 2059-2065.

[40] Wang, M.D., Yin, H., Landick, R., Gelles, J., Block, S.M., 1997. Stretching DNA with optical tweezers. Biophys. J. 72, 1335-1346.

[41] Wu, P., Kazys, R., Stepinski, T., 1997. Optimal selection of parameters for the angular spectrum approach to numerically evaluate acoustic fields. J. Acoust. Soc. Am. 101, 125-134.

[42] Xie, W., Cao, C., Lü, Y., Hong, Z., Wei, B., 2006. Acoustic method for levitation of small living animals. Appl. Phys. Lett. 89, 214102. 
[43] Xie, W., Cao, C., Lü, Y., Wei, B., 2002. Levitation of iridium and liquid mercury by ultrasound. Phys. Rev. lett. 89, 104304.

[44] Zhao, D., Thomas, J.L., Marchiano, R., 2019. Computation of the radiation force exerted by the acoustic tweezers using pressure field measurements. J. Acoust. Soc. Am. 146, 1650-1660. 